\title{
Zur Stimmung von Xylophonen in Malawi und Nordmoçambique Kritischer Forschungsbericht und Untersuchungen
}

\author{
von Florian Kragl
}

$-1-$

Das Xylophonspiel im Gebiet von Nordmoçambique und Malawi zählt nicht eben zu den häufig untersuchten Themenkreisen der Ethnomusikologie. Nur wenige Forschungen beschäftigen sich mit dieser besonderen Art, das Holmxylophon zu spielen, und alle stammen aus der Feder Gerhard Kubiks, dem auch umfassende Dokumentationen der Musik der Region auf Tonband und Film zu verdanken sind.

$$
-2-
$$

Allein: Die Aufmerksamkeit auf diese Musiktraditionen zu lenken, war nicht der ausschlaggebende Grund für vorliegende Studie. Vielmehr erweckten die Hypothesen Kubiks zur Stimmung der Xylophone dieses Raums mein Interesse. Kurz gefasst, basieren seine Ansätze auf der Annahme einer von der Obertonreihe abgeleiteten Stimmung. Doch genau eines jener Xylophone, das mangolongondo Waisoni Msusas, dessen Stimmung Kubik in einer seiner diesbezüglichen Veröffentlichungen mitteilte, passt in keiner Weise zu dieser Vermutung. Eine zuerst nur oberflächliche Untersuchung der Skala dieses Instruments zeigte mir, dass hier eher eine temperierte Hexatonik vorliegen dürfte: eine sehr ungewöhnliche Stimmung.

$$
-3-
$$

Es bot sich also an, diesen Widersprüchen im Rahmen einer umfassenderen Studie, wie sie hier nun vorliegt, näher nachzugehen. Dazu wird zuerst eine kurze allgemeine Einführung in den betreffenden Xylophonstil gegeben, sofern sich daraus Konsequenzen für die Deutung der Skalen der Instrumente ergeben. Es folgt eine Darstellung der Hypothesen Kubiks zur Stimmung der Xylophone, die von ihm in mehreren Arbeiten entwickelt und ausgebaut wurden, um anschließend daran Kritik zu üben und mögliche Schwachstellen der Argumentation aufzuzeigen. Dies wird die Basis legen für die Untersuchungen an insgesamt acht Instrumenten, deren zum Teil überraschende Ergebnisse das abschließende Resümee bilden werden.

\section{$-4-$}

Mein Dank gilt allen voran Herrn Prof. Dr. Gerhard Kubik, ohne dessen Forschungstätigkeit auf diesem Gebiet weder die nötigen Aufnahmen noch das beinahe ebenso wichtige Grundwissen vorhanden wären, um sich an ei- 
ne solche Untersuchung zu wagen. In mehreren Gesprächen förderte er diese Studie und gab wichtige gedankliche Hilfestellungen. Auch danke ich dem Phonogrammarchiv der Österreichischen Akademie der Wissenschaften, insbesondere Prof. Dr. Dietrich Schüller und Dr. Helmut Kowar für die Bereitstellung der benötigten Aufnahmen, deren Nutzung mir durch die freundliche Genehmigung von Prof. Dr. Gerhard Kubik ermöglicht wurde.

$-5-$

Ehe man sich der Stimmung der Xylophone im genannten Gebiet widmen kann, ist eine kurze Einführung in das Xylophonspiel dieses Bereichs vonnöten, und zwar aus zwei Gründen. ${ }^{1}$ Zum einen vermittelt diese den notwendigen allgemeinen Hintergrund, vor dem eine Spezialstudie erst erfolgen kann. Zum anderen ist das Wissen insbesondere um die Spielart und Struktur der Stücke eine der wesentlichen Grundlagen, auf denen Kubik seine hier in Diskussion gestellte These zur Stimmung der Xylophone errichtete.

$-6-$

Das Gebiet, in dem die hier behandelten Xylophontypen auftreten, wird gemeinhin als Nyasa/Ruvuma-Kulturraum umschrieben und umfasst das Gebiet um den Nyasa-See (oder: Malawi-See) und entlang des Ruvuma, also in erster Linie Malawi und Nordmoçambique sowie angrenzende Gebiete von Tanzania. Kubik unterscheidet in einer frühen Publikation (1964:79-80) drei Gebiete: das Gebiet südlich des Chilwa-Sees, die westliche Zentralregion und den Nordosten. Diese nach musikalischen Gesichtspunkten getroffene Unterscheidung - „Musical practices in these three areas are distinctly different " (Kubik 1964:80) - wird jedoch bereits in diesem Aufsatz für das Xylophon nur bedingt in Anwendung gebracht. Zwar unterscheidet Kubik 1964 das Xylophonspiel des Zentralraumes und des Nordostens, unterlässt aber in späteren Veröffentlichungen eine solche Trennung und spricht allgemein von der ,,südlichen Verbreitungszone des Holmxylophons in Ostafri$k a$ " (unter anderem Kubik 1982:160).

\section{$-7-$}

Die Bevölkerung des Nyasa/Ruvuma-Raums gliedert Kubik (1982:39-40) in vier Gruppen: 1. die Maravi-Gruppe mit dem Siedlungsgebiet westlich des Nyasa-Sees in großen Teilen des zentralen und südlichen Malawi, zu der unter anderem die -Chewa, -Mang'anja, -Nsenga, -Mbo, -Ntumba und -Zimba rechnen, 2. die -Tumbuka und -Tonga nördlich der Maravi-Gruppe,

Nach Kubik 1964; 1965; 1982:38-43, 158-163; 1987:31-46; 1989:10-11, 42-43; 2001. 
3. die -Ngonde zwischen North Rukuru und dem Songwe und die -Nyakyusa in Tanzania am Nordufer des Nyasa-Sees, 4. die Ruvuma-Völker, zu denen unter anderem die -Makonde, -Ndonde, -Makua, -Meto, -Shirima, -Lomwe und -Yao gehören. Die Trennung der ethnischen Gruppen insbesondere im Gebiet des unteren Ruvuma ist jedoch relativ unscharf, da sich beinahe jedes Dorf zu einer anderen ethnischen Gruppe zählt. Auch die musikalischen Unterschiede sind eher geringfügig: „Im Nyasa/Ruvuma-Kulturgebiet sind die nächsten musikalischen Verwandten der -Makonde die -Makua, -Ndonde, -Lomwe, -Shirima und -Meto " (Kubik 1982:43). Folglich stellen nur die -Yao eine gewisse Ausnahme dar.

$$
-8-
$$

Das Xylophonspiel ist besonders unter diesen Ruvuma-Völkern verbreitet, zu deren Gebiet auch der 'Zentralraum', besiedelt von Untergruppen der Makua wie den -Shirima, den -Lomwe und den -Meto, gerechnet werden muss. Der Xylophonstil ist im gesamten Nordmoçambique vertreten und wurde vermutlich von Chiyao oder Elomwe Sprechenden nach Malawi gebracht, im Falle der -Yao vermutlich im 18. und 19. Jahrhundert. Schließlich erreichte das Instrument auch die -Ngonde und die -Nyakyusa am nördlichen Ende des Nyasa-Sees (Kubik 1987:34). Wie lange diese Tradition des Xylophonspiels bereits besteht, ist schwer zu sagen. Jedenfalls aber dürfte es Teil einer Reihe erhaltener, älterer Musikformen sein, die nicht auf den in vielen Bereichen starken islamischen Einfluss zurückgehen (Kubik 1982:42).

$$
-9-
$$

Die Spieler der Xylophone sind meist Jungen im Alter von ca. 20 Jahren. Daneben wurden von Kubik auch ältere Expertenspieler aufgenommen, um die sich mitunter regelrechte Xylophonschulen gebildet haben. Selbst die Xylophonschule einer Spielerin für Mädchen und Frauen ist bekannt, wie generell das Xylophonspiel bei Frauen zwar selten, aber nicht unüblich ist. ${ }^{2}$ Das Xylophonspiel hat primär unterhaltende Funktion, wird aber von kleinen Jungen ab dem Alter von etwa zehn Jahren auch oft tagelang in Unterständen auf den Feldern zum Verjagen von Affen und Vögeln gespielt. In dieser Praxis wird wohl der Grund für die teilweise sehr hohe Perfektion des Spiels liegen. Möglicherweise besitzt das Xylophonspiel auch ein gemeinschaftsbildendes Element (dazu Kubik 1987:33; 2001:13) in dem Sinne, dass durch das gemeinsame Spiel Freundschaften zwischen Jungen geknüpft und gefestigt werden. In solchen Fällen könnte das Xylophon zumindest bedingt als Altersklasseninstrument verstanden werden.

Zum Spiel der Frauen sowie zu den Xylophonschulen siehe besonders Kubik 1987:33. 
Die Bezeichnungen für das Instrument variieren zwischen den verschiedenen ethnischen und auch Sprachgruppen. Es heißt mangwilo bei den -Shirima in Nordmoçambique, dimbila bei den -Makonde in Moçambique, mangolongondo bei den -Yao in Malawi, mambirira bei den -Lomwe in Nordmoçambique und, ohne Silbenduplikation, mambira ${ }^{3}$ bei den -Khokala im Raum des Chilwa-Sees in Malawi und Nordmoçambique (Kubik 1982:245-247). Mangolongondo leitet sich aus der onomatopoetischen Lautformel ngólóngòndò-ngóló-ngòndò für Patterns des Xylophons her, die Wurzeln -mbira oder -mbila benennen in Südostafrika entweder Lamellophone oder Xylophone und meinen wohl die Stäbe der Instrumente, $\mathrm{ma}$ - ist ein Kumulativpräfix, vermutlich im Sinne von 'viele Stäbe' (van Zanten 1980:108; Kubik 1987:33-34). Ungeklärt sind die Herleitung von mangwilo (Kubik 2001:9) und die Bedeutung des Präfix di- (bei den -Chopi: timbila).

$-11-$

Die Bauart der Xylophone (ausführlich Kubik 1982:160; 2001:10-12) weist ebenfalls leichte regionale Unterschiede auf, wenngleich hier die Gemeinsamkeiten zu überwiegen scheinen. Bei den Instrumenten handelt es sich durchgehend um Holmxylophone mit relativ wenigen Tasten, die auf zwei Bananenstämmen ruhen. Kleinere Xylophone, etwa jene der Alomwe, haben nur fünf bis sieben Platten, größere wie jene der Wayao bis zu zehn oder mehr (Kubik 1987:33). Allgemein sind die Instrumente der -Yao meist größer als diejenigen der -Shirima, die kleinsten Instrumente finden sich bei den -Makonde (Kubik 2001:9). Allen gemeinsam ist wiederum, dass oft nicht auf allen Platten gespielt wird. Gelegentlich dient eine Taste als Ersatz, oder es werden bei verschiedenen Stücken unterschiedliche (jeweils unvollständige) Kombinationen aus dem Gesamtvorrat ausgewählt. Auch die Anordnung der Tasten am Instrument variiert von Stück zu Stück, sodass die angestrebten musikalischen Strukturen in möglichst effizienter Weise in Bewegungsmuster umgesetzt sind. Auf den Bananenstämmen werden die Platten von kleinen Holzstäbchen voneinander getrennt, die in das Holz der Unterlage gesteckt sind. Bei den -Makonde (und -Cuabo) sind diese Stäbchen auf einer Seite nicht zwischen, sondern durch die Platten gesteckt (Kubik 2001:9); die Platten liegen zudem auf Grasbüschelunterlagen. Einen speziellen Resonator kennen die Xylophone nicht, Resonanz entsteht im Hohlraum zwischen den Bananenstämmen unter den Platten. In einem Fall (in der Nähe des ChilwaSees) wurde dieser Effekt verstärkt, indem vermutlich in entsprechender Absicht unter dem Xylophon ein Loch gegraben wurde (Kubik 1987:33).

Nicht zu verwechseln mit dem gleichnamigen Trogxylophon der -Cuabo. 
Am Spiel sind üblicherweise zwei einander gegenüber sitzende Spieler beteiligt, von denen jeder in beiden Händen einen Schlägel hält. Nur selten hilft ein dritter, dessen Aufgabe es ist, die verschobenen Platten wieder zurecht zu rücken. Komplexere Aufgabenverteilungen sind sehr selten. ${ }^{4}$ Einer der beiden Spieler, hinkünftig Spieler I, spielt eine unverändert wiederholte Grundreihe, in die der Spieler II einfällt und freiere, oft virtuose Variationen dazu entfaltet. Seine Aufgabe ist es auch, gegebenenfalls verschobene oder herausgesprungene Tasten wieder in die richtige Position zu bringen. Die Termini für die funktional unterscheidbaren Spieler variieren zwischen den verschiedenen ethnischen Gruppen. ${ }^{5}$ Die einzige grössere Abweichung von dieser Praxis besteht in dazu konträren Aussagen der jungen Xylophonspieler Dixon und Rosário, die Kubik 1962 mitteilten, das mangwilo hätte üblicherweise elf Tasten und würde in der Regel von vier Spielern (je zwei auf einer Seite) gespielt (Kubik 1964:91). Diese Angaben konnten jedoch weder bei Forschungen im näheren Umkreis der Jungen noch in anderen Gebieten des oben skizzierten Raumes verifiziert werden.

\section{$-13-$}

Die gespielten Stücke sind fast ausschließlich reine Instrumentalstücke, nur in seltenen Fällen wird dazu gesungen, wenngleich die Stücke vermutlich tonsprachliche und rhythmische Assoziationen auslösen und verbalisiert werden können, was wiederum für die Namensgebung der Stücke ausschlaggebend sein dürfte (Kubik 2001:12-13,39). Aussagen zur Struktur der Stücke (ausführlich Kubik 2001:32-47) wurden von Kubik anhand der Analyse von sieben Stücken entwickelt, die allesamt von den Spielern Venjiwa und Jenja innerhalb von zwei Tagen aufgenommen wurden. Weniger ausführliche Untersuchungen am Spiel Waisoni Msusas konnten als Bestätigung herangezogen werden (Kubik 1987:34, 37-41 mit einigen analytischen Bemerkungen und hauptsächlich Notenbeispielen; ebenso Kubik 1989:10-11, 42-43).

$$
-14-
$$

In den untersuchten Fällen dient eine Platte des Instruments als Bordun, als tonales Zentrum des Stücks. Tonale Wechsel kennt diese Musik nicht. Die Borduntaste wird bis auf Ausnahmen (eines der sieben untersuchten Stücke von Venjiwa und Jenja) durch ('duple-division') Interlocking von beiden Spielern geschlagen. In allen sieben Stücken ist dies die tiefste Platte der verwendeten Auswahl. Da bei einem Stück die tiefste Platte weggelassen

\footnotetext{
$4 \quad$ Vgl. als Beispiel Kubik 1989:10 und die Notizen zum Spiel Waisoni Msusas weiter unten.

5 Siehe dazu die Anmerkungen zu den untersuchten Aufnahmen.
} 
wird, übernimmt die nächsthöhere, nun die tiefste der Gebrauchsskala, die Bordun-Funktion. Der Bordun wird meist von der rechten Hand geschlagen. Die andere Hand spielt freiere Patterns, wobei jeder Spieler für sich eine polyrhythmische Formel schlägt. Die Spielfelder der beiden Spieler sind variabel, in keinem der von Kubik untersuchten Fälle spielt ein Spieler auf allen Platten. Im Detail erweist sich für die untersuchten Stücke eine hohe Komplexität der Kompositionstechnik: kanonartige Strukturen, 'Doppelschlagtechnik' durch Antizipation oder Verzögerung von Schlägen, verschiedene rhythmische Muster, mitunter sogar Time-line-Formeln etc. Ebenfalls auffällig und für Kubiks Hypothese zur Stimmung entscheidend sind häufige Sekundzusammenklänge, mitunter auch Sekundparallelen. Dies ist auch für die Vokalmusik der -Shirima, -Lomwe und anderer Gruppen des Makua-Clusters in Nordmoçambique charakteristisch und konnte zudem am mangolongondo-Spiel der -Yao beobachtet werden (Kubik 2001:48). Grundsätzlich kann jeder Ton zugleich mit jedem anderen der Skala angeschlagen werden. Es existieren keine verbotenen Dissonanzen oder dergleichen.

$-15$

Eine zentrale Position in den Untersuchungen Kubiks zum Xylophonspiel im Nyasa/Ruvuma-Raum nimmt die Frage nach der Stimmung der Instrumente ein. Analysen zur Struktur der Stücke stehen meist im Dienste dieser Problematik (so vor allem Kubik 2001). Nach welchen Vorstellungen, nach welchen Systemen ermitteln die Musiker die Tonhöhen — die im Nyasa/Ruvuma-Gebiet wohl interkulturell als Tongrößen verstanden werden ('klein' ist gleich 'hoch' usf.; vgl. zum Süden Malawis van Zanten 1980:110; zu Buganda Kubik 1992:65) —, auf die sie ihre Instrumente stimmen? Eine Frage, die lange Zeit ohne rechte Antwort blieb, bis Kubik in mehreren Arbeiten sukzessive eine These entwickelte, die wiederum fast ausschließlich auf Studien am Instrument von Venjiwa und Jenja beruht.

$$
-16-
$$

Gleiches gilt für den Vorgang des Stimmens. (Kubik konnte bei Venjiwa und Jenja den gesamten Vorgang der Herstellung eines Instruments zumindest in den wichtigsten Arbeitsschritten beobachten.) Aus einer großen Anzahl vorbereiteter Platten werden jene ausgewählt, die bereits ungefähr den Tonhöhen der intendierten Skala entsprechen. Feinkorrekturen werden durch Abhacken von Holz vorgenommen: im mittleren Bereich der Tasten an der Unterseite für tiefere Stimmung, am Rand der Platten an der Unterseite für höhere. Die Tonhöhen werden nach Stimmformeln und Stimmstücken 
(Kubik 2001:12) ermittelt bzw. kontrolliert, von denen jedoch m. W. keine Aufnahmen vorliegen.

$$
-17-
$$

Wesentlich schwieriger zu erfassen als diese praktischen Vorgänge sind die Skalen, nach denen gestimmt wird. Die einzige sofort zu erfassende Gemeinsamkeit aller von Kubik untersuchten Xylophone scheint in der Hexatonik zu liegen. Doch wie sind die Skalentöne im Oktavrahmen organisiert? In frühen Publikationen der 1960er Jahre beschäftigte sich Kubik (1964:92-93) erstmals mit diesem Themenkreis und entwickelte im Anschluss vor allem an A. M. Jones eine These, die von der Annahme einer unvollständigen Heptatonik ausgeht, bei der quasi ein Skalenton ausgelassen wird. Anlass zu dieser Vermutung gaben die auffälligen Entsprechungen bestimmter Intervalle beim Instrument von Venjiwa und Jenja. Die Skala kann als aus drei Standardintervallen A (181 Cents), B (214 Cents) und C (140 Cents) zusammengesetzt gedacht werden: 181 - 214 - 140 - [140 - 181] - 214 - 140 Cents. Die eckige Klammer markiert das Doppelintervall, der Oktavrahmen musste für die Hypothese künstlich geschlossen werden und wird nur einmal von einer realen Taste repräsentiert. Doch bleiben offene Fragen (Kubik 1964:93): Wie entsteht diese Skala? Gibt es sie auch anderswo? Warum fehlt ein Skalenton? Weshalb sind die Standardintervalle genau 140, 181 und 214 Cents groß? Welchem musikalischen, akustischen oder psychologischen Gesetz entspricht eine solche Skala?

$$
-18-
$$

Dieser Ansatz wurde aber nicht weiterverfolgt. Bereits in der nächsten Publikation zu diesem Thema deutete Kubik (1965:38-39) eine neue Hypothese an, zunächst ohne näher darauf einzugehen: Er verglich die Stimmung desselben Instruments mit der Obertonreihe eines harmonisch komplexen Klangs. Erst Mitte der 1980er Jahre griff er diese Vermutung in der bislang umfassendsten Studie zum Xylophonspiel dieses Gebiets wieder auf (Kubik 2001). Er ordnet darin den (meist) sechs Tönen der Gebrauchsleiter wiederum des Instruments von Venjiwa und Jenja die Partialtöne 8, 9, 10, 11, 13 und 15 der harmonischen Obertonreihe zu (Kubik 2001:22 mit Tabelle und graphischer Darstellung) und erläutert: ,Charakteristisch scheint, daß bis zum 11. Teilton die Reihe kontinuierlich ausgeweitet wird, während die Teiltöne 12 und 14 - beinahe als ob sie für das Klangempfinden der Ashirima-Musiker uninteressant wären, weil sie nur höhere Oktaven der 'banalen' Teiltöne 6 und 7 darstellen - vermieden werden" (Kubik 2001:23). Gestützt werden diese Annahmen vom zweiten berücksichtigten Instrument, jenem der Jungen Dixon, Rosário und Carlindo. Bei diesem Xylophon seien es die Teiltöne 7-13, die von den Xylophonplatten repräsentiert werden (Kubik 2001:23-24). Wie so hohe Teiltöne als Muster einer 
Stimmung verwendet und wie dieser Tonraum entdeckt werden konnte, ist heute nicht mehr rekonstruierbar. Allerdings: „Dass im südlichen Afrika gelegentlich sehr hohe Partialtöne willentlich verstärkt werden, ist durch verschiedene Autoren belegt, u.a. besitzt David Rycroft eine Aufnahme des Spielers einer europäischen Maultrommel durch einen Bantu-sprachigen Südafrikaner, bei der bis zum 11. Teilton verstärkt wird" (Kubik 2001:49).

$$
-19-
$$

Als Zwischenergebnis hält Kubik fest: „Nach den Messergebnissen dieser beiden Stimmungen scheint somit für die Shirima-Xylophone als Konstante postulierbar, daß sie in der Intervallik höhere Partialtonbereiche nachahmen, und als Variable, daß der spezifische Ausschnitt aus der Partialtonreihe, den eine bestimmte Stimmung repräsentiert, nicht immer gleich zu sein scheint" (Kubik 2001:24; Hervorhebungen original). In allen Fällen sei das Ergebnis eine hexatonische Gebrauchsskala, wobei jenen Tasten eine zentrale Bedeutung zukommt, welche die Teiltöne 7-9 wiedergeben.

„Diese drei Töne werden [...] im Sinne der Teiltonreihe als so sehr verwandt wahrgenommen, daß man sie beliebig auch zusammen anschlagen kann. Daher sind Sekunden von \pm 231 Cents (d.i. ein Zusammenklang von Tönen, die Partialton 7 und 8 repräsentieren) und von \pm 204 Cents (Partialton 8 und 9) ein so charakteristisches Merkmal der harmonischen Zusammenklänge beim mangwilo und wie wir es später in Malawi feststellten auch beim mangolongondo-Holmxylophon der -Yao" (Kubik 2001:25).

Die Interpretation dieser merkwürdigen Sekundzusammenklänge eröffnet nur zwei Wahlmöglichkeiten:

„entweder wir nehmen an, dass [sic!] dieses System völlig unharmonisch sei, also die Stimmung im Grunde zufällig ist und von sechs verschiedenen Tönen alle beliebig miteinander klingen können, oder aber wir nehmen an dass ein harmonisches System vorliegt dessen Komponenten alle auf ein tonales Zentrum bezogen sind, und daher jeder darin enthaltene Ton von diesem Zentrum ableitbar ist" (Kubik 2001:48; Hervorhebungen original).

$$
-20-
$$

Die Ergebnisse seiner Untersuchung zur Stimmung fasst Kubik in sieben Punkte (Kubik 2001:49-50; Hervorhebungen original): Erstens gibt es einen Bordunton, der zweitens dem 8. Partialton entspricht und somit Grundtoncharakter besitzt. Drittens kann (in einem der sieben analysierten Stücke) 
auch der nächsthöhere Teilton, der mit dem häufigeren Bordunton das charakteristische Sekundintervall bildet, als Bordun verwendet werden. Viertens werden niedrigere Partialtöne, insbesondere Verdopplungen des dritten Partialtons, vermieden, ,, als ob sie zu banal wären. “ Das Übergehen des 14. Partialtons dürfte in seiner Nähe zum 15. begründet liegen. Fünftens entsteht die Harmonik des Systems ,,über den zwei benachbarten und gleichzeitig einsetzbaren Borduntönen [...]. Teilton 8 und 9 können jederzeit zusammen angeschlagen werden, weil sie ja Teil desselben Systems sind, nämlich der Partialtonreihe. Deshalb bilden sie in diesem System auch keine 'Dissonanz'. " Folglich können — sechstens — alle Töne zusammen erklingen, wobei ,eventuell sich bildende Differenztöne beim gleichzeitigen Anschlagen von Klangstäben, die in einem Intervall wie Teilton 8 und 9 gestimmt sind, den Fundamentalton verstärken und damit den Bordunton." Sie ,, unterstützen daher die harmonisch-tonale Struktur dieser Stimmung. “ Schließlich werden - siebtens - auf den Platten für die Teiltöne 8 und 9 die Basisreihen, -formeln etc. gespielt, während sich auf den übrigen, höhere Partialtöne repräsentierenden Tasten ,,das Gewebe der übergelagerten und stets in neuen Variationen sich ergießenden Melodiegestalten abspielt".

$$
-21-
$$

Daraus folgt: „Alle harmonisch-tonalen Elemente, die wir in der mangwiloMusik festgestellt haben, sind in akustischen Gesetzen begründet" (Kubik 2001:50). Die harmonische Reihe sowie das Phänomen der Differenztöne erklären, weshalb alle Töne, besonders aber Sekunden, simultan angeschlagen werden können und welche Funktion der Bordun erfüllt, der (oktaviert) mit dem von allen Differenztönen verstärkten Fundamentalton identisch ist. „Das Ergebnis ist eine Musik, die im Grunde einen - wenn auch in feinste, melodisch-rhythmische Elemente polyphon aufgelösten - Simultanakkord der Partialtöne 8, 9, 10, 11, 13 und 15 darstellt" (Kubik 2001:50).

$$
-22-
$$

Die gegenwärtig letzte Studie zum Themenbereich (Kubik 1987:31-46), in die auch neues Forschungsmaterial aus Feldforschungen von 1983 einfloss, verfasste Kubik wenige Jahre nach der eben referierten ausführlichen Analyse des Spiels von Venjiwa und Jenja. Hinsichtlich der Stimmung gibt Kubik als Beleg seiner praktisch unveränderten Hypothese auch die Hertz- und Cents-Werte zum Xylophon von Waisoni Msusa (Kubik 1987:39). Die Werte wurden jedoch offensichtlich nicht auf ihre Kongruenz zur Hypothese überprüft, da sie von einer gedachten Obertonreihe massiv abweichen und eher eine äquidistante hexatonische Skala realisieren. Die in der vorangehenden Studie noch auf das mangwilo und mangolongondo, ethnisch also auf -Shirima und -Yao bezogenen Aussagen werden nun ohne weitere Belege für den gesamten Nyasa/Ruvuma-Raum generalisiert. „Tuning proce- 
dures of log xylophones in the Nyasa/Ruvuma culture area of Malawi and northern Moçambique follow some firmly established principles. " (Kubik 1987:35) Genannt werden -Yao, -Lomwe und -Shirima. Zum System der Stimmung:

„1. The xylophone tunings often duplicate higher partials of the natural harmonic series over a single fundamental, usually from the 7th to the 13th partials, sometimes even from the 8th to the 15th, omitting partials 12 to 14.

2. Since the pitches are based on a single fundamental [...] musical compositions for this kind of xylophone are marked by an ever present, bordun-tone which is produced with the xylophone player's own means; a note fades away fast on a log xylophone [...] so it has to be produced by the interlocking method. The bordun tone ist the tonal reference point of the music. Usually it appears on the lowest xylophone slat, or on the next to it [...].

3. Further, since the tunings are derived from the use of constituent notes of the harmonic scale, any two notes struck simultaneously may be acceptable as a consonant chord, and this includes the use of seconds, a prominent feature in this xylophone style. [...] This does not exclude, however, the simultaneous use of thirds, especially among Yao xylophone performers [...] [P] arallel seconds arise naturally from the simultaneous intonation of tones representing partials 8 plus 9 , proceeding to 9 plus 10, or even downwards to 7 plus 8 [...]. In the natural harmonic series, any two [neighbouring] notes sounded together also reinforce the single fundamental through difference tones " (Kubik 1987:35; Hervorhebungen original).

$-23-$

Nach den mehrfach modifizierten Hypothesen liegt hiermit quasi das Endergebnis der Studien vor. In Kurzform: 1. Die Tasten entsprechen den Partialtönen 7-13, mitunter auch 8-15, 12 und 14 werden übersprungen. 2. Ein Bordunton entspricht in fast allen Fällen dem oktavierten Grundton einer solchen harmonischen Reihe. 3. Durch Sekundparallelen entstehen Differenztöne, die wiederum den hypothetischen Grundton verstärken. ${ }^{6}$

6 Auf eine etwas unglückliche Formulierung sei hier nur hingewiesen: In einer allgemeinen Einleitung zum Xylophonspiel des Nyasa/Ruvuma-Gebiets notiert Kubik (1982:42): „,Nach den Erfahrungen unserer Forschungen in Nordmoçambique 1962 ist -Makonde-Musik meist hexatonisch mit Intervallen um 160 bis 180 Cents “. Doch keine Hexatonik kann durch Intervalle von 160-180 Cents entstehen, das durchschnittliche Intervall liegt bei 200 Cents. Entweder denkt Kubik hier an 
Eine gewisse Ergänzung zu diesen Arbeiten stellt ein Gespräch mit Gerhard Kubik dar, das ich am 9. November 2001 mit ihm führen konnte. Darin resümierte er spontan seine Sichtweise, machte jedoch gegenüber den obigen Aussagen gewisse Einschränkungen. Auch wurde dabei klar, dass die Ergebnisse aus seiner Sicht nach wie vor zumindest teilweise den Status von Hypothesen besitzen: apodiktische Konstatierungen und Verallgemeinerungen wie besonders in der Studie von 1987 wurden vermieden.

$-25-$

Die Einschränkungen bestehen insbesondere in einer klaren Scheidung verschiedener Gebiete, an der sich auch die vorliegende Studie orientiert. Denn während für die Region der Mitukwe-Berge in Nordmoçambique für die 1960er Jahre, aus denen die frühen Aufnahmen stammen, mit hoher Sicherheit eine gewisse kulturelle Abgeschiedenheit vor allem gegenüber dem Rundfunk angenommen werden kann, trifft dies für das Ruvuma-Tal sowie für Malawi nicht zwingend zu. Der Geltungsbereich der Thesen beschränkt sich demnach - wie auch die Detailstudien - auf die Bergregion in Nordmoçambique vor einer möglichen Einflussnahme durch Medien und Rundfunk (insbesondere Radio). Im Ruvuma-Gebiet waren dagegen bereits bei Kubiks erster Forschungsreise 1962 Einflüsse durch Radio und islamische Musik zu verzeichnen (Kubik 1964:94). ,Europäischer, vor allem portugiesischer Einfluß kam schließlich durch koloniale Institutionen während der portugiesischen Herrschaft (bis 1974) in das Gebiet der-Makonde: aus der Musikpraxis der christlichen Missionen, der Schulen und des Rundfunks, vor allem der gut zu empfangenden Station Nampula (Nordmoçambique) und Dar es Salaam " (Kubik 1982:43). In Malawi wiederum ist ein sehr starker islamischer Einfluss zu verspüren, der sich auf das Tonsystem ausgewirkt haben könnte.

Abgesehen von der Frage, ob überhaupt alle Xylophone nach der von Kubik postulierten Systematik gestimmt sind - das von Waisoni Msusa zeigt, wie erwähnt, markante Abweichungen - ; abgesehen also von solchen konkreten, nur durch Untersuchungen an einzelnen Instrumenten bzw. von einzel-

eine unvollständige Heptatonik mit einem Doppelintervall oder an die harmonische Stimmung. In beiden Fällen aber wäre ein entsprechender Hinweis zum Verständnis der Stelle nötig gewesen. 
nen Aufnahmen zu klärenden Problemen, scheint gegenüber Kubiks Argumentation in mehrfacher Hinsicht Kritik angebracht.

$-27-$

Zunächst erweckt die Methodik gewisse Bedenken. Die Stimmung der Xylophone wurde bei den alten Aufnahmen (Xylophon von Venjiwa und Jenja sowie von Dixon, Rosário und Carlindo) von A. M. Jones mit einem Stroboconn abgenommen, bei den Aufnahmen des Xylophons von Waisoni Msusa aus den 1980er Jahren mit einem Korg Chromatic Tuner WT-12. Dies ist in zweifacher Hinsicht problematisch. Zum einen hätte es, da die Aufnahmen ohnehin zugänglich sind, kaum eines Mehraufwandes bedurft, die Teiltoncharakteristik der Xylophontasten durch exaktere Verfahren der Schallverarbeitung und -analyse in gemittelten Spektren festzustellen, was insbesondere im Falle der Stroboconn-Messungen ein wesentlich zuverlässigeres Ergebnis geliefert hätte. Zum anderen wurde nicht danach gefragt, ob diese physikalischen Messergebnisse auch den auditiven Eindrücken entsprechen, die von den Xylophontasten hervorgerufen werden. Denkbar wären etwa ein mehrdeutiges Tonhöhenempfinden oder eine charakteristische Verschiebung der Tonhöhe gegenüber dem physikalischen Frequenzwert. ${ }^{7}$

$$
-28-
$$

Ähnlich unpräzise erfolgte offenbar die Ermittlung der Centswerte. ${ }^{8}$ Anstatt sie nach der unproblematischen Formel $1200 * \log _{2}\left(\mathrm{f} / \mathrm{f}_{0}\right)$ für die einzelnen Xylophontasten mit Referenz auf eine ausgewählte Taste (wohl den Bordunton) zu errechnen, wurden anscheinend Werte aus einer Umrechnungstabelle verwendet und dann erst sekundär durch Addition und Subtraktion die Verhältnisse zwischen den Tasten erfasst. Die bei den meisten Tabellen Kubiks ebenso angeführten abgelesenen Tabellenwerte zeigen, dass der Referenzwert der Tabelle ca. 170 bzw. 340 bzw. 680 usf. (die Oktavlage ist unbedeutend) betragen haben muss. Das eigentliche Problem liegt nun darin, dass durch dieses Ablesen aus vorgefertigten Werten ein gewisser Messbzw. Rechenfehler entsteht, da nur Näherungswerte zur Verfügung stehen. Die Abweichungen zu den exakten Werten schwanken zwischen 0 und 5 Cents. (Man vergleiche die Abweichungen bei den einzelnen Instrumenten unten.)

$$
-29-
$$

Unüblich sind schließlich die negativen Centswerte in einer einzigen Tabelle (Kubik 2001:23), da auch Kubik von der Oktave als Rahmenintervall wenn es auch auf den kleinen Xylophonen real nicht vorhanden sein muss ausgeht. (Von dieser Annahme zeugen etwa Begriffe wie 'hexatonisch', die

Zur gesamten Problematik siehe Schmidhofer 1994.

Vgl. die Tabellen bei Kubik 1982:158; 1987:35, 39; 2001:18, 23. 
nur vor dem Konzept der Oktavspanne verwendet werden können.) Statt negativer Zahlen müsste also eine Bereinigung der Oktavlage durchgeführt werden, um vergleichbare und aussagekräftige Werte zu erhalten, da davon ausgegangen werden muss, dass sich in höheren und tieferen Oktaven die Skala wiederholt. Hinweise für eine davon abweichende Annahme werden nirgends erwähnt.

$$
-30-
$$

Allerdings darf nicht übersehen werden, dass diese Kritik am methodischen Vorgehen keineswegs die Hypothese Kubiks zu Fall bringen wird. Sie soll nur hellhörig machen für mögliche Fehlerquellen auch im Hinblick auf die nachfolgenden Studien an den Aufnahmen. Doch finden sich neben diesen 'Lappalien' auch ungleich tiefer greifende Ungereimtheiten. Dies trifft unter anderem für die These des Borduntons als oktaviertem Grundton der Skala zu. Zwar steht außer Zweifel, dass die untersuchten Musikstücke eine Art Bordun verwenden. Dessen Funktion als Grundton der Skala ist jedoch allem Anschein nach nicht von besonderer Bedeutung, da er in dieser Rolle auch von anderen Tönen der Skala abgelöst werden kann. Die Vorstellung von einer Art ausdifferenziertem Klang, der doch letztlich 'Simultanakkord' ist, gerät dadurch massiv ins Wanken, da dieses eine der sieben untersuchten Stücke ohne Bordunton (ist gleich oktavierter Grundton) einer völlig anderen Struktur unterliegt. Wie sehr auch ein nicht geschlagener Grundton durch Differenztöne gegeben sein mag: im Gehörseindruck wird letztlich doch der in diesem Fall 9. Teilton dominant sein und — wenn überhaupt — wesentlich schwächer ein hypothetischer Grundton.

$$
-31-
$$

Dies lässt bereits an der Hypothese zweifeln, denn sie scheint in ihrer obigen Form manches Wesentliche des Xylophonspiels und der Xylophonstimmug außer Acht zu lassen und lediglich als Ausnahme zu tolerieren. Geradezu unwahrscheinlich erscheint die These indes, wenn man nach der möglichen Entstehung einer solchen Skala zu fragen beginnt. Immerhin lautet die Prämisse, dass hier Teile der harmonischen Reihe bis zum 15. Partialton genützt werden. Wie kann ein menschliches Ohr diese Töne als Tonhöheneindrücke wahrnehmen? Der Verweis auf Belege einer Nutzung der harmonischen Reihe bis einschließlich des 11. Partialtons krankt — abgesehen vom Fehlen einer zitierfähigen Untersuchung - vor allem daran, dass dadurch über die Teiltöne 12-15 eben nichts ausgesagt ist.

$$
-32-
$$

Forschungen zu den Fähigkeiten der auditiven Wahrnehmung des Menschen sprechen eher gegen die Möglichkeit, diese immens hohen Komponenten der harmonischen Obertonreihe noch bewusst als Tonhöhen (oder -größen) wahrnehmen zu können. Sowohl bei der menschlichen Stimme - Sonder- 
fälle wie der Singformant in europäischer 'Kunstmusik' ausgenommen als auch bei Instrumenten (besonders Chordophonen), bei denen so hohe Teiltöne zumindest physikalisch eindeutig verifizierbar sind, haben diese hohen Teiltonkomponenten nur noch einen geringen Anteil an der Gesamtenergie des harmonischen Klangs und sind, nicht zuletzt aufgrund gegenseitiger Maskierung der Komponenten im Klang, meist schlichtweg zu leise, als dass sie neben den ungleich stärkeren tieferen Teiltönen als selbständige Empfindungen wahrgenommen werden könnten. Die geschätzte Häufigkeit, mit der die Teiltöne 15 und 16 von Vokalklängen mit ihren Spektraltonhöhen wahrnehmbar sind, tendiert gegen Null (Terhardt 1998:323, Abb. 11.8). Doch noch entscheidender mag sein, dass diese Komponenten üblicherweise in Frequenzbereichen liegen - bei einem Grundton von beispielsweise 220 $\mathrm{Hz}$, dem a, liegt der 10. Partialton bei $2200 \mathrm{~Hz}\left(\right.$ ca. ein cis ${ }^{4}$ ) -, die vom Ohr zwar wahrgenommen, jedoch insbesondere beim simultanen Erklingen mit anderen Klangkomponenten nicht mehr als diskrete Tonhöhen aufgelöst werden. (Die Fähigkeit zur Unterscheidung von Tonhöhen lässt etwa ab $2000 \mathrm{~Hz}$ rapide nach [vgl. Terhardt 1998:261, Abb. 9.19].) Als Teile eines harmonischen Klangs haben diese hohen Frequenzbereiche vor allem eine Funktion hinsichtlich der Klangfarbe des Schallereignisses und nur einen geringen Einfluss auf tonale Empfindungen (vgl. Terhardt 1998:349-351). Kurzum: Die bewusste Verarbeitung der Spektraltonhöhen hoher Teiltöne ist möglich, aber wenig wahrscheinlich.

\section{$-33-$}

Vor diesem Hintergrund scheint es auch wenig angebracht, von 'banalen' Teiltönen zu sprechen. Jeder, der einmal versucht hat, aus nicht übermäßig manipulierten Klängen auch nur die unteren Partialtöne herauszuhören, wird wissen, wie wenig 'banal' die Aufgabe ist, die Partialtöne 3-7 gehörsmäßig von einem harmonischen Klang zu scheiden. $\mathrm{Zu}$ diesen allgemeinen Bedenken gegenüber einer Wertung der harmonischen Reihe nach dem Kriterium 'Komplexität ist gleich Rang in der Reihe' treten konkrete Widersprüche in der Darstellung. So darf etwa der 7. Teilton vorkommen, doch die Teiltöne 12 und 14 sind als 'banal' ausgenommen. Übersehen wird, dass bei oktavbereinigten Skalen wie in vorliegendem Fall solche Platten, die nach dem 7. Partialton gestimmt sind, nicht von solchen zu unterscheiden sind, die dem 14. Teilton entsprechen: der einzige Unterschied zwischen 7. und 14. Teilton liegt in der von Kubik (zurecht) unberücksichtigt gelassenen Oktavlage. Wie kann dann der 14. Teilton 'banal' sein, während der 7. bei gewissen Gebrauchsskalen zusammen mit dem 8. und 9. die charakteristischen Sekundintervalle bildet und geradezu konstitutives Merkmal des Stils und in weiterer Folge der postulierten Stimmung ist? Und schließlich wäre nach 
solchen Kriterien der in den Skalen vertretene 10. Teilton als Oktavierung des 5. eine geradezu unterträgliche Plattitüde.

$-34-$

Die zentrale Frage wurde allerdings bisher kaum berührt (und wird auch von Kubik nur am Rande behandelt): Wie groß dürfen die Abweichungen von der idealen Skala sein? Zur Beantwortung dieser Frage bieten sich für das untersuchte Xylophonspiel mehrere Kriterien an. Zunächst impliziert die These von Differenztönen, die einen konstanten Grundtoneindruck entstehen lassen, Rückschlüsse auf die Toleranzspanne der Stimmung. Tatsächlich entspricht die Tonhöhe eines Differenztons von der Art $f_{2}-f_{1}$ bei $f_{2}>f_{1}$ dem Grundton einer harmonischen Reihe, wenn die beiden Teiltonfrequenzen benachbarte harmonische Komponenten dieser Reihe sind: also etwa der 7. und 8. oder der 8. und 9. Teilton. Allerdings gilt dies nur bei so gut wie exakten Frequenzverhältnissen. Sobald einer der beiden oder beide Teiltöne nur leicht verstimmt sind, weicht ihre Frequenzdifferenz von der Grundtonhöhe und der Differenzton vom Grundton der gedachten harmonischen Reihe $a b$.

\section{$-35-$}

Ein Beispiel aus dem Kern der Arbeiten Kubiks möge dies verdeutlichen: Beim Xylophon von Venjiwa und Jenja haben die beiden tieferen Tasten die (aufgrund abweichender Bandgeschwindigkeiten bei der Aufnahme korrigierten) Frequenzwerte 461 und $512 \mathrm{~Hz}$, was laut Kubik einem Intervall von 181 Cents (tatsächlich gerundeten 182 Cents) entspricht (Kubik 2001:18). Kubik bringt dieses mit dem (gerundet) 204 Cents großen Intervall zwischen dem 8. und 9. Partialton der Naturtonreihe zusammen (Kubik 2001:22). Dies ergäbe bei einem 8. Teilton mit $461 \mathrm{~Hz}$ einen 9. mit (gerundet) $519 \mathrm{~Hz}$. Der Grundton der harmonischen Reihe wäre damit 519-461 ist gleich $58 \mathrm{~Hz}$; ein sehr tiefer Ton, entsprechend ungefähr dem $B_{1}$ der Kontraoktave. Der Differenzton zwischen den Platten des Xylophons dagegen ergibt 512-461 ist gleich $51 \mathrm{~Hz}$, was in etwa dem $\mathrm{As}_{1}$ entspricht. Der Unterschied beträgt (gerundet) $223 \mathrm{~Hz}$, mehr als einen Ganzton der europäischen Musik! Von einem durch Differenztöne verstärkten Grundtoneindruck kann also gar keine Rede sein, die Abweichung liegt weit oberhalb jeder denkbaren Unterschiedsschwelle (dazu weiter unten). Ihr Intervall übertrifft sogar manche der Intervalle zwischen den Xylophonplatten. Abweichungen von nur wenigen Hertz bei den gestimmten Tasten führen aufgrund der tiefen Lage des angenommenen Grundtons bei den Differenztönen zu extremen CentsAbweichungen, da eine gleiche Hertz-Differenz eine ungleich höhere CentsAbweichung in der tiefen Lage bedeutet. 
Ebenso gegen die Annahme von konstitutiven Differenztönen spricht die Eigenheit von Kombinationstönen gerader Ordnung (zu denen der Differenzton $\mathrm{f}_{2}-\mathrm{f}_{1}$ rechnet), erst bei relativ hohen Schallpegeln hörbar zu werden (Terhardt 1998:238, 429-430; vgl. Hall 1997:394-397). Die Erfüllung dieser Voraussetzung bei kleinen, nicht speziell resonierten Holmxylophonen ist zweifelhaft. Zudem sind Xylophonklänge per se äußerst flüchtig, die Intensität der Klänge schwindet sehr rasch. Das gewichtigste Argument gegen die Annahme von Differenztönen jedoch ist, dass man sie schlichtweg nicht hört. Weder die Originalprotokolle Kubiks zu seinen Aufnahmen (Kubik $1962 ; 1967 ; 1983 / 84)$ noch seine publizierten Arbeiten handeln über merkwürdige, nicht geschlagene Tonhöhen, wie sie durch Differenztöne entstehen. Selbst bei sehr hohen Wiedergabepegeln konnte auch ich keine Kombinationstöne irgendwelcher Art bei den Aufnahmen feststellen und hörte lediglich die - in meiner subjektiven Apperzeption - faszinierenden Sekundzusammenklänge.

$$
-37-
$$

Als Substitut für diese Differenzton-These könnte eine durch die einzelnen Teiltöne hervorgerufene virtuelle Tonhöhe ${ }^{9}$ dienen, die wiederum den Grundton repräsentieren würde. Dagegen spricht jedoch erneut, dass diese Tonhöhe schlichtweg nicht hörbar ist. Dies verwundert wenig, da einige der konstitutiven Voraussetzungen zum Entstehen einer solchen Tonhöhenempfindung fehlen: exakte Simultaneität unter den Teiltönen, einander zeitlich entsprechende Phasen der einzelnen Komponenten (Einschwingvorgang, quasi-stationärer Teil, Ausschwingvorgang), hohe Homogenität zwischen den verschiedenen Simultanklängen usf.

$$
-38-
$$

Mit der Zurückweisung der Annahme einer von Differenztönen evozierten oder forcierten Grundtonhöhe geht auch das erste Kriterium einer Bestimmung der Toleranzspanne verloren. Eine weitere, zweite Möglichkeit, der Frage nach den tolerierten Abweichungen von einer Musterskala zu begegnen, besteht in einem Blick in bisherige Forschungen zu dieser und anderen Regionen des Xylophonspiels in Afrika. Zum Ausmaß der tolerablen Vari-

9 Die Theorie der virtuellen Tonhöhe, entwickelt von Ernst Terhardt seit den frühen 1970er Jahren, besitzt bis heute die größte Erklärungspotenz für das Entstehen eines einheitlichen Tonhöheneindrucks bei harmonisch komplexen Klängen, bei Residua etc. Ausführlich dazu mit den entsprechenden Verweisen Terhardt 1998:345368. Ältere Auffassungen, nach denen der Grundtoneindruck bei Residua (harmonischen Reihen ohne physikalische Repräsentation der Grundtonhöhe) durch Differenztöne hervorgerufen werde, gelten längst als veraltet (Hall 1997:396). 
anzen liegen in Bezug auf den Kulturraum, aus dem das hier untersuchte Stück stammt, m. W. kaum eingehende Studien vor. Kubik gibt zwar an: „, There is a margin of tolerance of up to \pm 20 Cents in xylophone tunings of the Nyasa/Ruvuma culture area, as we were able to access through comparison of the tunings of a dozen recorded instruments. " (Kubik 1987:37). Doch er nennt hier weder das ideale Schema, von dem aus die Abweichungen gerechnet werden - vermutlich denkt er an die Stimmung nach der Obertonreihe -, noch gibt er konkrete Werte als Belege an. Angesichts der in mancherlei Hinsicht problematischen Thesen zum Tonsystem der Xylophone erscheinen auch diese Angaben zur Abweichung zu einem guten Teil zweifelhaft. Das Instrument von Waisoni Msusa etwa, dessen Stimmung Kubik 1987:39 angibt, wurde offenbar nicht in die Untersuchungen zum Tonsystem eingebunden. Es kann folglich auch nicht als Vergleichswert dienen. Und schließlich käme es einem Zirkelschluss gleich, die Toleranzspanne aus den Xylophonstimmungen im Vergleich zur Skala zu ermitteln und anschlließend die Stimmungshypothese mit dem Argument der Toleranzspanne zu verifizieren. Ich nenne als Orientierungshilfe (zumindest für ihren jeweiligen Objektbereich) aussagekräftigere Ergebnisse zu anderen afrikanischen Xylophonstilen.

$$
-39-
$$

Kubik erwähnt etwa zu Xylophonmusik, die er 1968 in Buganda aufgenommen hat, bezüglich der Ungenauigkeit seiner Messmethode von \pm 5 Cents: „This figure ist acceptable in the comparative study of African tunings, and well within the margin of tolerance of present-day musicians in southern Uganda" (Kubik 1992:63). Vermutlich könnte diese Aussage auch ohne ihre Beschränkung auf afrikanische Musiker bzw. Tonsysteme gelten, bedenkt man, dass die Frequenzunterschiedsschwelle für Sinustöne - und die Tonhöhenempfindung bei Xylophonklängen ist in hohem Maß von einzelnen Teiltönen abhängig - unter $500 \mathrm{~Hz}$ bei ca. $1 \mathrm{~Hz}$ liegt, für Sinustöne darüber im Absolutwert leicht ansteigt und schließlich ab einer Referenzfrequenz von ca. $1000 \mathrm{~Hz}$ etwa 0,2\% beträgt (Terhardt 1998:261). Als Richtwerte einige Umrechnungen in das Cents-Maß (zuerst Frequenzwerte, dann CentsZahlen): 101:100 - 17,2; 201:200 - 8,6; 301/300 - 5,8; 401:400 - 4,3; 501:500 - 3,5; 0,2\% Frequenzdifferenz - 3,5. Die 5 Cents Toleranzspanne entsprechen also in etwa dem psychophysikalischen Modell, das Unabhängigkeit von kulturellen Faktoren beanspruchen kann (Terhardt 1998:261).

$$
-40-
$$

Kubiks für den Xylophonstil in Buganda angenommenes Modell einer äquidistanten Pentatonik nun umfasst beim untersuchten Instrument reale Tonschritte zwischen 190 und 279 Cents (Kubik 1992:64f.), impliziert also eine Irrelevanzschwelle von bis zu 50 Cents (einem Viertelton)! Wim van Zanten 
untersuchte 1970 im Süden Malawis bei den Asena verschiedene Instrumente, darunter Xylophone mit gut 20 Stäben namens valimba bzw. ulimba bzw. malimba (van Zanten 1980:107). Die Tonhöhen ermittelte er im Vergleich mit 54 Stimmgabeln (gestimmt im 4-Hz-Abstand) im Selbstversuch unter Absprache mit den Musikern (zu diesem Verfahren auch Schmidhofer 1994:332). Sein Bestreben galt hauptsächlich dem Nachweis oder der Widerlegung der Annahme einer äquidistanten heptatonischen Stimmung. Das Ergebnis — „It has been pointed out that the tuning model may be slightly different from the equidistant heptatonic scale model." (van Zanten 1980:118) - bringt zwar trotz oder gerade wegen methodologischer Exaktheit nicht die gewünschte Eindeutigkeit. Doch die Annahme einer temperierten Heptatonik erscheint zumindest einigermaßen als Hintergrundfolie der Stimmung gesichert, wobei ebenfalls Abweichungen der einzelnen Tasten von bis zu 50 Cents anzunehmen sind.

$-41-$

Solchen Resultaten entgegen stehen Untersuchungen wie jene, die Vincent Dehoux und Frédéric Voisin um 1990 in Zentralafrika durchführten. Im Gegensatz zu den Messwerten bei Kubik und van Zanten (und auch bei vorliegender Studie) können sie auf experimentellen Ergebnissen bauen, bei denen die betreffenden Musiker die gewünschte Tonhöhe auf einem Synthesizer einzustellen gebeten wurden. Wie sehr die Musiker und ihre Reaktionen von dieser allerdings ungewöhnlichen Versuchssituation beeinflusst waren, sei dahingestellt. Als Ergebnis resultierten jedenfalls drei verschiedene Intervalle mit ca. 200, 240 und 285 Cents, wobei gilt: ,the margin of tolerance of these intervals is more or less 15 cents " (Dehoux/Voisin 1993:18; erweiterte und modifizierte Studie als Dehoux/Voisin 1992 in dem hier betrachteten Aspekt identisch). Dies kann jedoch nicht als Gegenbeweis zu Kubik und van Zanten gelten. Vielmehr liegt hier ein völlig anderes, nicht äquidistantes Tonsystem vor, Analogieschlüsse sind durch keine Indizien berechtigt. Die Künstlichkeit einer äquidistanten Stimmung begründet geradezu die größeren Differenzen zwischen den einzelnen Tasten.

$$
-42-
$$

Will man aber für den Ruvuma-Kulturraum an Kubiks Hypothese einer aus der Obertonreihe deduzierten Skala festhalten, sollte die Toleranzgrenze für Abweichungen vom genauen Wert wohl eher niedrig angesetzt werden, da sich in solchem Fall die Intervalle aus quasi 'natürlichen' und, so die Annahme, hörbaren Tonhöhendistanzen herleiten. Man hat damit also ein drittes Kriterium zur Ermittlung der tolerierbaren Abweichungen zur Hand. Da ein direkter Tonhöhenvergleich - beispielsweise zu gesungenen Vokalen - möglich ist, kann als Toleranzspanne die Unterschiedsschwelle von Sinustönen gelten, zumal die Teiltöne ohnehin Sinustoncharakter besitzen und 
auch Xylophontasten aus dem betreffenden Gebiet hinsichtlich ihrer tonalen Charakteristik im Wesentlichen von einem einzelnen Teilton (ist gleich Grundton) bestimmt sind (siehe dazu unten).

Die Unterschiedsschwellen von Sinustönen, die bereits ungefähr in ihrer Frequenzabhängigkeit beschrieben wurden, sind jedoch auch von der Signaldauer in dem Sinne abhängig, dass der eben wahrnehmbare Frequenzunterschied JNFD (just-noticeable frequency difference) mit abnehmender Dauer der Töne zunimmt. Eine Verkürzung der Dauer um den Faktor 10 entspricht grob einer Zunahme der JNFD (in Bark) um denselben Faktor (Zwicker/Fastl 1990:117; Terhardt 1998:261-262). Einige ungefähre Beispielwerte für die Dauer von 100 bzw. 50 (Klammerausdrücke) ms nach dem Muster: Testtonfrequenz: JNFD: 125 Hz: ca. 30 (50) Cents; $250 \mathrm{~Hz}$ : ca. 10 (20) Cents; 500 Hz: ca. 7 (10) Cents; 1000 Hz: ca. 5 (7) Cents (alle Werte so genau als möglich abgelesen aus Zwicker/Fastl 1990:116, Fig. 5.14; Prozentwerte wurden in Cents-Zahlen umgerechnet). Da die Grundtöne der Xylophone im Bereich von ca. 250 bis $950 \mathrm{~Hz}$ liegen und Xylophonklänge zudem nicht die Eindeutigkeit von Sinustönen besitzen, kann für die bei starkem Anschlag etwa 50 bis 100 ms deutlich präsenten tonalen Anteile der Xylophonklänge (siehe unten) eine Unterschiedsschwelle von etwa 10 bis 20 Cents angenommen werden.

Überblickt man nun die obige Diskussion der früheren Forschung, kann man sich dem Eindruck nicht entziehen, dass in den genannten Arbeiten implizit der Versuch unternommen wurde, kulturabhängige Skalen durch eine unmittelbare Ableitung aus akustischen 'Gesetzen' quasi zu rechtfertigen, wie dies im 19. Jahrhundert verschiedentlich für die abendländische Diatonik versucht wurde. Ganz gleich, wie sehr diese Versuche hinsichtlich der Kongruenz von Skala und akustischem Phänomen haltbar sind, tragen diese Ambitionen stets zweierlei prinzipielle Fehlerquellen. Erstens verraten sie ein Bestreben, eine Musik durch Verbindungen zu vermeintlich klaren und letztgültig fassbaren, dadurch auch leicht $\mathrm{zu}$ handhabenden naturwissenschaftlichen Erkenntnissen sowohl besser zu verstehen als auch in gewisser Hinsicht zu rechtfertigen. Bei Kubik etwa klingt in manchen Passagen die postulierte Komplexität der Stimmung als Wertungskriterium ('banale' Teiltöne) an.

$$
-45-
$$

Zweitens, daraus folgend, gehen diese Studien zumindest teilweise an ihrem Gegenstand vorbei, indem sehr starre vorgefasste Prinzipien auf die betrachtete Musik projiziert werden: im vorliegenden Fall besonders das Phänomen der Differenztöne und des kontinuierlichen Grundtoneindrucks. Die- 
se Vermutungen beruhen auf unscharfen Reinterpretationen einer Skala nach akustischen und psychoakustischen Erscheinungen und sind an der Musik selbst nicht verifizierbar (nicht hörbar). Vielmehr sind die Ansätze permanent in Gefahr, an der Musik vorbei zu argumentieren, da die Musik in ein vorgefertigtes System eingezwängt wird. Dieses wurde freilich klug gewählt: je höher man in der Partialtonreihe fortschreitet, desto mehr Möglichkeiten einer Zuordnung bieten sich (aufgrund der kleiner werdenden CentsAbstände bei gleichen Hertz-Abständen) an und desto mehr potentielle Tonhöhen sind erschlossen (wie auch Kubik 2001:49-50 anmerkt). Die Teiltonreihe ist unendlich fortsetzbar - doch nur mathematisch, nicht empirisch!

$$
\text { - } 46 \text { - }
$$

Fazit: Ausgeschlossen werden kann, dass Differenztöne dazu dienen, einen Grundtoneindruck zu erzeugen. Die auffälligen Sekundzusammenklänge müssen anders erklärt werden. Sie verlangen auch in keiner Weise nach einer psychophysikalischen Grundlage, denn ob Zusammenklänge als konsonant oder dissonant empfunden werden, ist maßgeblich vom Erwartungsund Erfahrungshorizont des Hörers abhängig. Niemand käme auf die Idee, im Rahmen einer harmonischen Analyse eines Werks europäischer Musik Akkorde als unmittelbare Realisation von Obertonklängen oder dergleichen $\mathrm{zu}$ deuten. Warum sollte nicht beim Xylophonspiel im Nyasa/RuvumaGebiet ebenso von komplexen Konventionen des Musikmachens ausgegangen werden, die auf jahrhundertelangen Entwicklungen von Hörgewohnheiten beruhen?

$$
-47-
$$

Mit der These der Differenztöne verliert auch jene vom konstanten Grundtoneindruck an Gewicht. Zweifellos ist ein Bordunton das tonale Zentrum der untersuchten Stücke, aber ein tiefer, irgendwie hervorgerufener Grundtoneindruck kann wohl ausgeschlossen werden. Keinesfalls entsteht bei den Stücken eine Art 'Simultanakkord' oder zumindest nicht mehr und nicht weniger als bei anderer schneller Xylophonmusik wie etwa bei den äquidistant gestimmten Xylophonen in Buganda. Allenfalls kann eine Skala angenommen werden, die sich, auf welchen Wegen auch immer, von der harmonischen Partialtonreihe ableitet, obgleich diese These angesichts der sehr hohen Teiltöne zumindest fragwürdig erscheint. Jedenfalls wird diese Zuordnung von den Musikern heute nicht mehr getroffen und erkannt, da in solchem Fall die Abweichungen von der idealen Skala weitaus geringer ausfallen müssten (siehe die Untersuchungen zu den einzelnen Instrumenten unten). Und letztlich ist es keine Besonderheit, wenn eine Skala nach dem Muster der Teiltonreihe gebildet ist. So gut wie jede musikalische Skala basiert entweder auf harmonischen Reihen oder auf irgendwie praktisch ermittelten Teilungen der Oktave (Teilung einer Saite, Unterscheidungen nach Größe 
oder Gewicht eines Idiophons usf.). Außergewöhnlich wäre beim Xylophonspiel des Nyasa/Ruvuma-Gebiets lediglich das bewusste Spielen mit Differenztönen, einer virtuellen Tonhöhe oder dergleichen und dem daraus folgenden Grundtoneindruck. Doch eben diese Merkmale sind nach den bisherigen Ausführungen mehr als unwahrscheinlich.

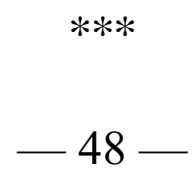

Für die Untersuchungen wurden Aufnahmen herangezogen, die Kubik bei seinen Forschungsaufenthalten 1962 und 1983/84 im Nyasa/Ruvuma-Gebiet machte. ${ }^{10}$ Das wichtigste Kriterium für die Auswahl war eine separate Aufnahme der Stimmung; - eine Extraktion der einzelnen Platten aus einem gesamten Stück, sei es durch Methoden der Signalverarbeitung oder durch 'Heraushören', hätte eine zu große Fehlerquelle dargestellt. Folglich konnte die Aufnahme des Xylophonspiels von Señora Muhua und eines 10 Jahre alten Jungen in einem Dorf in den Mitukwe-Bergen bei Nova Freixo (heute Cuamba), die Kubik 1962 durchführte, nicht berücksichtigt werden. Eine weitere Einschränkung ergab sich aus den obigen Ausführungen zum Xylophonstil des Nyasa/Ruvuma-Gebiets. Es interessierten nur solche Aufnahmen, die tatsächlich aus diesem Raum stammten, wobei hier die Zugehörigkeit der Spieler zu einer ethnischen Gruppe als primäres Auswahlkriterium herangezogen wurde. Und schließlich ergab sich eine dritte Beschränkung finanzieller Art, da ausschließlich in Wien archivierte Aufnahmen miteinbezogen werden konnten.

$$
-49-
$$

Damit beschränken sich die anschließenden Studien auf insgesamt acht Instrumente, die Kubik 1962 und 1983 aufnehmen konnte. Nicht berücksichtigt sind die im Ethnologischen Museum Berlin archivierten Aufnahmen von 1967 bei den -Lomwe, -Yao, -Khokola und -Ngonde in Malawi (vgl. Kubik 1982:160); Aufnahmen von 1976 bei den -Nyakyusa in Tanzania; verstreute Aufnahmen Kubiks in Malawi seit 1980, archiviert in Wien und Berlin; Aufnahmen aus Malawi von Moya Aliya Malamusi, archiviert in Mainz bei Wolfgang Bender; und das Material von Hugh Tracey und Margot Dias. ${ }^{11}$

$$
-50-
$$

$\mathrm{Zu}$ den Aufnahmen der Skalen der Xylophone ist zu bemerken, dass bei den älteren Aufnahmen von 1962 (B 8995, B 9088, B 9029, B 9038, B 9145) die

10 Die Aufnahmenummern wurden aus der Archivierung des Phonogrammarchivs übernommen.

11 Für diese Angaben danke ich Gerhard Kubik. 
Platten in sehr rascher Folge angeschlagen wurden; vermutlich deshalb, um die Stimmung mit einem Stroboconn abnehmen zu können (so geschehen bei B 8995 und B 9088). Dazu war ein quasi-kontinuierlicher Klang notwendig. Bei den neueren Aufnahmen von 1983 (B 28278, B 28330, B 28347) dagegen sind die Platten einfach angeschlagen, jede Platte in drei- bis vierfacher Wiederholung.

$$
-51-
$$

Die Untersuchung der Instrumente erfolgte in drei Etappen. Zunächst galt es, die Skala des jeweiligen Instruments zu ermitteln, was wiederum in zwei Schritten geschah. Ausgehend von der Problematik der früher üblichen messtechnischen Verfahren (dazu Schmidhofer 1994: 327-329) orientierte ich mich zunächst an Sinuston-Vergleichen der Xylophontöne im Selbstversuch. Die Vergleichstöne generierte ich mit Emil Lubeijs Programm TestSnd, Version 1.0. Beim Selbstversuch wiederholte ich die Wiedergabe der Aufnahme sowie den Vergleich mit einem Sinuston beliebige Male, bis ich hinsichtlich einer auditiven Entsprechung der Tonhöhenempfindungen hinlängliche Gewissheit erlangte. Der Sinuston wurde vor, nach oder zugleich mit dem aufgenommenen Xylophonklang gespielt. Meine Aufmerksamkeit galt jeweils nur dem augenblicklich zu vergleichenden Sinuston, die Skala als Gesamtkonzept wurde bewusst vernachlässigt. Sie hätte als quasi Melodielinie meine Urteilsfähigkeit maßgeblich verwirren können.

$$
-52-
$$

Die so ermittelten Werte wurden in einem zweiten Schritt mit Amplitudenspektren der Klänge verglichen. Dabei bediente ich mich des Programms S_Tools-STx (Version 2.14), entwickelt am Institut für Schallforschung der Österreichischen Akademie der Wissenschaften unter der Leitung von Werner A. Deutsch. Die Fast Fourier Transformation wurde über die Dauer von $5 \mathrm{~s}$ gerechnet, was einer Genauigkeit der Frequenzwerte von weit unter $1 \mathrm{~Hz}$ entspricht. Diese Mittelung der Signale konnte vorgenommen werden, da die Abnahme von kürzeren Amplitudenspektren keinerlei Hinweis erbrachte, dass sich die Frequenzwerte der einzelnen tonalen Komponenten der Xylophontasten über die Zeit ändern.

$$
-53-
$$

Sowohl bei den Sinuston-Vergleichen als auch bei der physikalischen Messung der Signale wurden die Frequenzwerte auf $1 \mathrm{~Hz}$ genau ermittelt bzw. gerundet. Dies liegt weit unter jeder denkbaren Unterschiedsschwelle, eine größere Genauigkeit hätte die Werte lediglich um Redundanzen bereichert. Überschritten die Spitzen von einzelnen Komponenten der Klänge die Bandbreite von $1 \mathrm{~Hz}$, wurde der Mittelwert zu weiteren Berechnungen herangezogen (dazu siehe unten). Eine Korrektur der Hz-Werte, wie sie eventuell aufgrund allfälliger Variation der Bandgeschwindigkeiten bei Aufnah- 
me und Wiedergabe nötig gewesen wäre, wurde nicht vorgenommen, da nach gegenwärtigem Wissensstand nicht von einer absoluten Stimmung auszugehen ist, geringere Abweichungen der absoluten Tonhöhen also vernachlässigbar sind (Kubik 1964:92). Dies bestätigt der gegenseitige Vergleich der einzelnen Xylophone, deren Tasten hinsichtlich der absoluten Tonhöhen mitunter erheblich voneinander abweichen. Als relevant sind die relativen Tonabstände der von den einzelnen Platten repräsentierten Tonhöhen anzusehen.

$$
-54-
$$

Der zweite Teil der Untersuchungen konzentrierte sich auf die Spieltechnik, die auf die einzelnen Xylophone angewandt wurde. Zwar scheint die Beziehung zwischen der Struktur der Musikstücke und der Stimmung der Instrumente weniger eng, als von Kubik angenommen. Die Musik völlig zu vernachlässigen hieße indes, diese Vermutung ohne stichhaltigen Grund zur Gewissheit zu erheben. Es wurde also jeweils zumindest e i n ausgeführtes Stück wenigstens gehörsmäßig grob analysiert, um besonders auffällige Abweichungen vom oben dargestellten Stil verzeichnen zu können. Geachtet wurde besonders auf das Vorhandensein einer Bordunplatte, das Interlocking in dem Sinne, dass die Angaben in den Protokollen zu den Spielbereichen der Spieler berücksichtigt wurden, die funktionale Unterscheidung von Spieler I und II und die Bauart der Instrumente. Besonders für das Ermitteln des Borduntons sowie der Sekundzusammenklänge konnten mit großem Gewinn Spektrogramme verwendet werden, die sich - sofern die Frequenz der einzelnen Xylophonplatten einmal bekannt war - nach einer Gewöhnungsphase beinahe wie ein Notentext lesen ließen.

$$
-55-
$$

Der letzte Punkt der Untersuchung galt schließlich der Interpretation des Beobachteten vor allem hinsichtlich einer Deutung der Skala als Realisation eines abstrakten, idealen Musters. Ausgehend von obigen Ausführungen wurden für jedes Instrument drei Interpretationsmuster erprobt: 1. die harmonische Reihe; 2. äquidistante Stimmungen; 3. verschiedene Standardintervalle, die zu einer Skala zusammengesetzt werden können. Die Toleranzspannen für diese drei Modelle sind unterschiedlich. Für eine von der Obertonreihe abgeleitete Stimmung, derer sich die Musiker entweder noch bewusst sind oder die in der Struktur der Musik verankert ist, können nur geringe Abweichungen als tolerabel gelten, die im Wesentlichen der Unterschiedsschwelle (bis zu 20 Cents) entsprechen. Gleiches gilt für die Hypothese verschiedener Standardintervalle, da bei größeren Toleranzspannen keine distinktiven Klassen voneinander geschieden werden könnten. Demgegenüber können nach den Ergebnissen zu anderen afrikanischen Xylo- 
phonstilen bei äquidistanten Stimmungen durchaus Abweichungen bis zu 50 Cents vorkommen.

$$
-56-
$$

Bei all diesen Versuchen sollte man sich freilich stets bewusst sein, dass die getroffenen Aussagen nie in dem absoluten Sinne von: 'die Skalen der Xylophone sind von der Obertonreihe abgeleitet' oder dergleichen gelten können. Vielmehr sind sie — hier wie bei jeder anderen Untersuchung — vom zuvor beschrittenen Erkenntnisweg abhängig, Aussagen wie die eben angeführte sind lediglich als verkürzte Form des Sprachgebrauchs sinnvoll. Jede Fragestellung determiniert die Aussageweite ihrer Antwort. Und demnach sind die Schlussfolgerungen dieser Untersuchung primär von drei Kriterien abhängig: von den Vergleichsfolien idealer Skalen, den dazu geschätzten Toleranzintervallen und der Festsetzung: je kleiner die Abweichung, desto besser. Die Ergebnisse sind also die eines Vergleichs der realen Skalen mit idealen $\mathrm{Mu}-$ stern und wollen, werden und können nicht das 'Wesen' der Stimmungen ergründen, sondern diese nach den erwähnten Konventionen beschreiben (vgl. die allgemeinen Bemerkungen bei Kragl 2001:77-85).

$*$

$-57-$

Bevor man sich den einzelnen Instrumenten zuwendet, stellt sich die Frage, welche Werte zum Vergleich mit den idealen Skalen oder Intervallen herangezogen werden: jene der Sinuston-Vergleiche oder jene aus den Amplitudenspektren? Für erstere spricht, dass sie zumindest einer menschlichen Wahrnehmung entsprechen; gegen sie, dass die Beschränkung auf nur eine Versuchsperson jeder Verallgemeinerung entgegen steht. Demgegenüber bieten die physikalischen Messungen exakte und objektivierbare Werte, die jedoch an sich überhaupt keine Wahrnehmung beschreiben können. Zur Entscheidung, welches Übel hier geringer wiegt, bietet sich ein Vergleich der beiden Messmethoden an.

$$
-58-
$$

Da die Werte der Messungen den nachfolgenden Tabellen zu den einzelnen Instrumenten entnommen werden können, erspare ich mir hier eine weitere Zusammenstellung. Es wird reichen, die statistischen Verhältnisse kurz zu beschreiben (alle Werte auf zwei Kommastellen gerundet). Dabei ergibt sich, dass die Werte der Sinuston-Vergleiche mit einem Mittelwert von 1,39 Cents von den physikalischen Messungen abweichen, wobei die Standardabweichung 9,77 Cents beträgt. Die vier Maxima liegen bei knapp über 20 Cents, das Maximum bei -22,18 Cents bei Platte 3 der Aufnahme B 9029. Es kann also keine systematische Abweichung der Sinuston- 
Vergleiche mit den physikalischen Messwerten ausgemacht werden. Die von Schmidhofer 1994:330 festgestellte Divergenz zwischen gemessenen und an 36 Versuchspersonen ermittelten Tonhöhen konnte ich bei den studierten Xylophonen nicht beobachten. Die Abweichungen bewegen sich weitgehend innerhalb der Toleranzspanne. Für weitere Berechnungen und Vergleiche konnten daher die mit $S \_$Tools ermittelten Werte verwendet werden, da sie nicht an eine individuelle Wahrnehmung gebunden sind und nichtsdestotrotz einen brauchbaren Eindruck von den Tonhöhen der Xylophontasten vermitteln.

$$
-59-
$$

Problematisch bleiben die älteren Messverfahren, insbesondere die Messungen mithilfe eines Stroboconns. Hier zeigt sich eine mittlere Abweichung gegenüber den $S_{-}$Tools-Werten von 2,84 Cents mit einer Standardabweichung von 9,22 Cents. Das Maximum liegt bei 21,34 Cents Abweichung für Platte 5 der Aufnahme B 8995. Die Stroboconn-Werte sind folglich im Vergleich zum physikalischen Signal ähnlich ungenau wie die Sinuston-Vergleiche, jedoch mit dem entscheidenden Nachteil, dass hier weder das Signal noch eine Empfindungsgröße beschrieben wird. Die Vorbehalte gegenüber dem Korg Chromatic Tuner WT-12 müssen dagegen aufgegeben werden, seine Messergebnisse entsprechen weitgehend jenen von $S \_T o o l s$. Der Mittelwert der Abweichungen liegt bei 0,00 Cents, die Standardabweichung bei 2,02 Cents. Die Abweichungen bewegen sich im Rahmen von \pm 3 Cents, lediglich bei Platte 9 der Aufnahme B 28330 beträgt die Abweichung $-4,38$ Cents.

$$
-60-
$$

Generell ist zu den Xylophonplatten zu bemerken, dass sie in der Regel ca. 200-300 ms klingen (wahrnehmbar sind), in seltenen Fällen noch länger oder auch kürzer, wobei praktisch in allen Fällen die oberen Teiltöne vor der Hälfte der Gesamtdauer in die Ebene des indifferenten Grundrauschens verschwinden. Der zeitliche Verlauf eines 200 ms langen Xylophonklangs kann in etwa folgendermaßen gefasst werden: In den ersten $30 \mathrm{~ms}$ steht eine breitbandige Anregung aller Frequenzen. Danach bilden sich einzelne, meist zwei bis vier Teiltöne heraus, die mit Ausnahme des untersten Teiltons innerhalb von 50-80 ms verklingen. Die erste, unterste Klangkomponente dominiert den restlichen Klang, der allerdings bereits sehr schwach (aber noch wahrnehmbar) ist. Freilich ist mit dieser Umschreibung nur ein Mittelmaß getroffen. In manchen Fällen ist die breitbandige Anregung länger oder kürzer, vor allem die Dauer des zweiten Teiltons scheint sehr variabel. Bei Klängen, die kürzer oder länger als $200 \mathrm{~ms}$ andauern, verändern sich diese relativen Phasen entsprechend. 
Die Spitzen der Klangkomponenten in den Amplitudenspektren sind zumeist auf eine Bandbreite von $1 \mathrm{~Hz}$ beschränkt. Ausnahmen bieten seltene Fälle, bei denen entweder die Spitze breiter als $1 \mathrm{~Hz}$ ist oder verschiedene Anschläge unterschiedliche Spitzen hervorbringen. Allerdings sind auch diese Ausnahmen an eine Variation von wenigen Hertz gebunden. Bei entsprechenden Platten wird in den Anmerkungen zu den einzelnen Xylophonen darauf hingewiesen. In die Tabellen wurden die Mittelwerte aufgenommen.

$$
-62-
$$

Fast immer ist eine eindeutige Tonhöhenempfindung gegeben. Die bei der Schallanalyse im gemittelten Amplitudenspektrum begegnenden mehrdeutigen Amplitudenspektren mit mehreren Teiltönen widersprechen einer unzweideutigen Tonhöhenwahrnehmung keineswegs, da die oberen Teiltöne um ein Vielfaches rascher verklingen als die unterste, meist durchgehend stärkste Klangkomponente (ähnlich Schmidhofer 1994:331). Bork (1986:401-402) bestätigt dieses Ausklingverhalten von Xylophonplatten an Instrumenten allerdings der westlichen 'Kunstmusik' (besonders Abb. 2 auf S. 402). Dass bei diesen Xylophonen die dritte Komponente des Klangs trotz der kurzen Ausklingzeit aufgrund des günstigen Strahlungswiderstandes den subjektiven Klangeindruck relativ stark beeinflussen kann, sollte nicht ohne weiteres auf außereuropäische Xylophone übertragen werden; insbesondere nicht auf die hier untersuchten Instrumente, da bei diesen eine dritte Komponente nicht nur zeitlich, sondern auch hinsichtlich der Schallintensität oft kaum vorhanden ist.

$$
-63-
$$

Ausnahmen bilden einige Platten, bei denen ich eine mehrdeutige Tonhöhenempfindung beobachten konnte. Meist war zum Hören der zweiten Komponente eine bewusste Konzentration auf obere Frequenzbereiche vonnöten, doch bei wenigen Tasten schwankte - bei isolierter Darbietung ohne den Kontext der übrigen Platten — die Wahrnehmung tatsächlich zwischen zwei ungefähr gleichwertigen Möglichkeiten. Insbesondere bei sehr tief gestimmten Platten war die zweite Komponente des Klangs oft sehr deutlich zu hören. Konkret handelt es sich um folgende Tasten (alle Werte in Hz): B 28278, Platte 8: 255/767 Hz; B 28330, Platten 2-4: 240/862, 271/953, 310/1041; B 28347, Platten 5-9: 217/642, 314/883, 300/806, 280/786, 255/696. Kursivsetzungen markieren solche Fälle, in denen die zweite Komponente auch physikalisch energiereicher (um 2-5 $\mathrm{dB}^{12}$ ) als die erste, untere ist. Eine merkwürdige Situation bietet B 9038, Platte 5 mit 645/1688 Hz.

12 Im gesamten Aufsatz immer dB (SPL). 
Hier ist die zweite Komponente stärker als die erste und trotzdem kaum zu hören.

$$
-64-
$$

Die Erklärung für diese scheinbar widersprüchlichen Beobachtungen wird wohl am ehesten das psychophysikalische Phänomen geben, dass für die Identifikation von Silben und Tonhöhen ein dominanter Frequenzbereich von ca. $700 \mathrm{~Hz}$ ausschlaggebend ist (Terhardt 1998:349-350). Je weiter sich Signale oder Signalkomponenten davon entfernen, desto weniger beeinflussen sie die Tonhöhenwahrnehmung von Klängen. Für die Bestimmung der Skalen jedenfalls wurde stets die tiefste Klangkomponente herangezogen, die durchweg auch zumindest gleich laut wie eine zweite, meist aber lauter empfunden wurde. Zudem bedingte der Kontext der übrigen Platten in den Xylophonstücken eine Konzentration auf den jeweils unteren Bereich, da bei keinem Xylophon alle Platten starke zweite Komponenten aufzuweisen hatten.

$$
-65-
$$

Die Stimmung jenes Instruments, auf dem die meisten seiner Aussagen zum Xylophonstil der Region beruhen, nahm Kubik am 14. Oktober 1962 in einem Dorf in der Nähe von Nova Freixo (heute Cuamba) in den MitukweBergen auf. ${ }^{13}$ Es ist das Instrument der damals 19-20 Jahre alten Xylophonspieler Venjiwa (Spieler II) und Jenja (Spieler I), die vermutlich zum Stamm der Ashirima (oder der Alomwe, einem Unterstamm der Amakua) zählen (vgl. Kubik 1962 und Kubik 1964:92; Kubik 2001:12 ordnet sie den -Shirima zu). Venjiwa ist der wakulela 'der Antwortende, der Respondierende', Jenja der opachera 'der Beginnende'. Die Aufnahme umfasst ein Instrumentalstück sowie die Skala des 7-stäbigen mangwilo. Zum Spiel werden nur 6 Platten verwendet, eine dient als Reserve. Die Anordnung der Stäbe wird von Stück zu Stück variiert.

$$
-66-
$$

Die Stimmung wird von Kubik in mehreren Publikationen mitgeteilt, zuerst Kubik 1964:92: 604,3 - 481 - 534 - 603,6 - 653 - 784 - 887 Hz (Angaben nach einer Stroboconn-Messung, die von A. M. Jones durchgeführt wurde). Später wurden die Werte korrigiert, da das Aufnahmegerät vermutlich um 74 Cents zu langsam lief. Außerdem wurden die Cents-Zahlen (hier Klam-

13 Die allgemeinen Anmerkungen zu den Aufnahmen wurden, wo nicht anders angegeben, den entsprechenden Originalprotokollen Kubiks (1962; 1983/84) entnommen, wie sie im Phonogrammarchiv den Aufnahmenummern beigeordnet sind. 
merausdrücke) beigegeben: 579 (394) - 752 (846) - 512 (181) - 461 (0) 579 (394) - 625 (529) - 850 Hz (1062 Cents) (Kubik 1987:35; 2001:18; Ausschnitt der Skala bei Kubik 1982:158). Die Klangstäbe werden in der Reihenfolge 850 - 752 - 625 - 579 - 512 - 461 den Partialtönen 8, 9, 10, 11, 13, 15 zugeordnet (Kubik 2001:22 mit Tabelle und graphischer Darstellung). Die Ergebnisse meiner Untersuchungen zur Skala sind in folgender Tabelle dargestellt.

Tab. 1: Stimmung des mangwilo von Venjiwa und Jenja (B 8995)

\begin{tabular}{|c|c|c|c|c|c|c|c|}
\hline \multicolumn{2}{|c|}{ Bordunton/Referenzton: } & \multicolumn{2}{|c|}{$478,00 \mathrm{~Hz}$} & \multirow[b]{2}{*}{5} & \multirow[b]{2}{*}{6} & & \multirow[b]{2}{*}{1} \\
\hline Platten & 2 & 3 & 4 & & & & \\
\hline $\begin{array}{l}\text { Kubik 1964:92 } \\
(\mathrm{Hz})\end{array}$ & 481,00 & 534,00 & 603,60 & 653,00 & 784,00 & 881,00 & 604,30 \\
\hline Sin.-Vgl. (Hz) & 476,00 & 533,00 & 598,00 & 644,00 & 773,00 & 874,00 & 603,00 \\
\hline S_Tools $(\mathrm{Hz})$ & 478,00 & 532,00 & 601,00 & 645,00 & 776,00 & 878,00 & 604,00 \\
\hline Cents & 0,00 & 185,30 & 396,43 & 518,75 & 838,86 & 1052,65 & 405,05 \\
\hline Intervalle $(\mathrm{Hz})$ & 54,00 & 69,00 & 44,00 & 131,00 & 102,00 & & \\
\hline Intervalle (Cents) & 185,30 & 211,13 & 122,32 & 320,11 & 213,80 & & \\
\hline \multicolumn{2}{|c|}{$\begin{array}{l}\text { OT-Reihe mit Grund- } \\
\text { ton: }\end{array}$} & 59,75 & $\mathbf{H z}$ & $(=478 / 8)$ & & & \\
\hline Teiltöne (Nr.) & 8,00 & 9,00 & 10,00 & 11,00 & 13,00 & 15,00 & 10,00 \\
\hline Teiltöne (Hz) & 478,00 & 537,75 & 597,50 & 657,25 & 776,75 & 896,25 & 597,50 \\
\hline Teiltöne (Cents) & 0,00 & 203,91 & 386,31 & 551,32 & 840,53 & 1088,27 & 386,31 \\
\hline Differenz $(\mathrm{Hz})$ & 0,00 & $-5,75$ & 3,50 & $-12,25$ & $-0,75$ & $-18,25$ & 6,50 \\
\hline Differenz (Cents) & 0,00 & $-18,61$ & 10,11 & $-32,57$ & $-1,67$ & $-35,62$ & 18,73 \\
\hline Statistik $(\mathrm{Hz})$ & $\mathrm{Mw}=$ & 57,14 & $s^{2}=$ & 85,16 & $\mathrm{~s}=$ & 9,23 & \\
\hline \multicolumn{2}{|c|}{$\begin{array}{l}\text { Temperatur mit Inter- } \\
\text { vall: }\end{array}$} & 200,00 & Cents & (Hexaton & & & \\
\hline Teiltöne $(\mathrm{Hz})$ & 478,00 & 536,54 & 602,24 & 675,99 & 758,78 & 851,70 & 602,24 \\
\hline Teiltöne (Cents) & 0,00 & 200,00 & 400,00 & 600,00 & 800,00 & 1000,00 & 400,00 \\
\hline Differenz (Hz) & 0,00 & $-4,54$ & $-1,24$ & $-30,99$ & 17,22 & 26,30 & 1,76 \\
\hline Differenz (Cents) & 0,00 & $-14,70$ & $-3,57$ & $-81,25$ & 38,86 & 52,65 & 5,05 \\
\hline Statistik (Cents) & $\mathrm{Mw}=$ & 210,53 & $\mathrm{~s}^{2}=$ & 4086,21 & $\mathrm{~S}=$ & 63,92 & \\
\hline
\end{tabular}

$-67-$

Einge allgemeine Erläuterungen zur Tabelle (sie gelten auch für künftige Tabellen zu anderen Xylophonen): Die Tabellen geben die Stimmungen für die Platten der Xylophone nach (wenn vorhanden) Kubik, den SinustonVergleichen und der Messung an den gemittelten Amplitudenspektren. Die Reihung der Spalten erfolgt der Größe der Hertz-Zahlen nach aufsteigend, die Plattennummern richten sich nach der Reihenfolge, in welcher die Platten bei der untersuchten Aufnahme angeschlagen worden sind. Kursivsetzungen markieren manuell eingefügte Werte gegenüber den sonstigen berechneten Werten. Die Cents-Zahlen für die einzelnen Platten sind in Bezug 
auf den Bordunton errechnet. Alle Werte sind auf zwei Kommastellen gerundet.

$$
-68-
$$

Beim Vergleich mit der Obertonreihe wurde anhand der Hertz-Intervalle zwischen den Platten ein Grundton geschätzt und - in diesem Fall — als Teiler des Borduntons definiert. Bei diesem Xylophon konnte den Ausführungen bei Kubik (siehe oben) gefolgt werden. Einen ungefähren Eindruck von den internen Abweichungen der realen Skala sollen Mittelwert (Mw), Varianz $\left(\mathrm{s}^{2}\right)$ und Standardabweichung (s) geben. ${ }^{14}$ Sie sind in $\mathrm{Hz}$ gerechnet, weil die Basis für den Vergleich mit der Obertonreihe die Hertz-Schritte sind.

$$
-69-
$$

Das genaue Negativ dazu bietet die dritte Sektion der Tabelle: der Vergleich mit einer temperierten Stimmung. Hier sind nun die Cents-Schritte der ausschlaggebende Faktor, entsprechend wird die Statistik in Cents ermittelt. Ansonsten erfolgen die beiden Vergleiche analog. Für den Vergleich mit einer Temperatur wurde - ausgehend von der Annahme, dass sich die Skala in der nächsten Oktave wiederholt - die Oktave durch die Anzahl der Intervalle geteilt, wobei auch der Mittelwert der Intervalle zwischen den Tasten berücksichtigt wurde. Als Beispiel: Wäre der Mittelwert ca. 200 Cents und nur die Taste 7 nicht vorhanden, wäre ein Doppelschritt anzunehmen gewesen.

$$
-70-
$$

Zur Deutung von Tab. 1: Platte 1 ist abgesetzt, da sie nicht benützt wurde und stets als Reserveplatte neben dem Xylophon lag. Als Bordunton ist eindeutig Platte 2 auszumachen, die charakteristischen Sekunden entstehen zwischen den Platten 2 und $3(54 \mathrm{~Hz})$ sowie 3 und $4(69 \mathrm{~Hz})$. Die potentiellen Differenztöne unterscheiden sich um $15 \mathrm{~Hz}$, was in dieser Lage (gerundet) 424 Cents, in europäischer Terminologie: einer übergroßen Terz entspricht! Dies wären in etwa zwei Intervallschritte auf der Skala. Selbst wenn diese Differenztöne gehört würden: dem Grundton entsprächen sie sicherlich nicht.

$$
-71-
$$

Dieser Grundton einer hypothetischen harmonischen Reihe müsste wohl mit $59,75 \mathrm{~Hz}$ - einer Oktavierung des Borduntons - angenommen werden. Allerdings sind die Abweichungen relativ groß. (Für die Berechnung der Varianz und Standardabweichung bei der Obertonreihe wurden die Doppelschritte vom 11. auf den 13. und weiter auf den 15. Teilton nur einmal berücksichtigt.) In zwei Fällen liegen sie weit über dem anzunehmenden Tole-

14 Varianz und Standardabweichungen sind stets mit Biaskorrektur gerechnet. 
ranzmaß, zweifellos im wahrnehmbaren Bereich (32,57 und 35,62 Cents, jeweils zu tief). Noch größer fallen indes die Abweichungen von einer temperierten Hexatonik aus (einmal 81,25 Cents zu tief), wenngleich zu bemerken ist, dass hier eine wesentlich größere Toleranzspanne zu unterstellen ist. Ungleich geringer wären die Abweichungen von drei Standardintervallen mit (ca.) 120, 185 und 210 Cents. Sie liegen in allen Fällen um bzw. unter 5 Cents: unter der Unterschiedsschwelle für gehaltene Sinustöne!

Wenige Tage zuvor, am 9. Oktober 1962, nahm Kubik vor der Missão Mitukwe am Fuße des Bergmassivs ein weiteres 7-stäbiges mangwilo auf, das ihm später (Kubik 2001) als Verifikation seiner Thesen zur Stimmung der Instrumente diente. Es wurde von den Missionsschülern Dixon, Rosário $^{15}$ und Carlindo (hier Dixon und Rosário) gespielt. Sie waren etwa 18-20 Jahre alt und gehörten zum Stamm der Alomwe. Die Aufnahme umfasst das Stück Napili ‘das Rebhuhn’ und die Skala des Xylophons.

$$
-73-
$$

Die Stimmung wurde wiederum mit dem Stroboconn abgenommen und diesmal um 76 Cents nach unten korrigiert: 581 (864) - 480 (531) - 441 (387) - 396 (200) - 353 (0) - 309 (-231) - 275,5 Hz (429 Cents). Die tiefste, siebte Platte wird laut Kubik beim Spiel nicht benutzt. Die Skala wird dem Bereich vom 7. bis zum 13. Teilton der harmonischen Reihe beigeordnet, der 12. Teilton wird übersprungen (alles nach Kubik 2001:24).

Tab. 2: Stimmung des mangwilo von Dixon, Rosário und Carlindo (B 9088)

Bordunton/Referenzton: $325,00 \mathrm{~Hz}$

\begin{tabular}{|c|c|c|c|c|c|c|c|}
\hline Platten & 3 & 2 & 4 & 5 & 6 & 1 & 7 \\
\hline $\begin{array}{l}\text { Kubik 2001:23 } \\
(\mathrm{Hz})\end{array}$ & 309,00 & 353,00 & 396,00 & 441,00 & 480,00 & 581,00 & 275,50 \\
\hline Kubik -76 Cents & 322,87 & 368,84 & 413,77 & 460,79 & 501,54 & 607,07 & 287,86 \\
\hline Sin.-Vgl. (Hz) & 321,00 & 370,00 & 413,00 & 460,00 & 501,00 & 612,00 & 287,00 \\
\hline S_Tools $(\mathbf{H z})$ & 325,00 & 370,00 & 413,00 & 463,00 & 504,00 & 607,00 & 288,00 \\
\hline Cents & 0,00 & 224,50 & 414,84 & 612,69 & 759,58 & 1081,51 & 990,75 \\
\hline Intervalle $(\mathrm{Hz})$ & 45,00 & 43,00 & 50,00 & 41,00 & 103,00 & & \\
\hline Intervalle (Cents) & 224,50 & 190,34 & 197,84 & 146,89 & 321,93 & & \\
\hline \multicolumn{2}{|c|}{ OT-Reihe mit Grundton: } & 46,43 & $\mathbf{H z}$ & $(=325 / 7$ & & & \\
\hline Teiltöne (Nr.) & 7,00 & 8,00 & 9,00 & 10,00 & 11,00 & 13,00 & 6,00 \\
\hline Teiltöne (Hz) & 325,00 & 371,43 & 417,86 & 464,29 & 510,71 & 603,57 & 278,57 \\
\hline Teiltöne (Cents) & 0,00 & 231,17 & 435,08 & 617,49 & 782,49 & 1071,70 & 933,13 \\
\hline Differenz $(\mathrm{Hz})$ & 0,00 & $-1,43$ & $-4,86$ & $-1,29$ & $-6,71$ & 3,43 & 9,43 \\
\hline Differenz (Cents) & 0,00 & $-6,67$ & $-20,24$ & $-4,80$ & $-22,91$ & 9,81 & 57,63 \\
\hline Statistik $(\mathrm{Hz})$ & $\mathrm{Mw}=$ & 47,00 & $\mathrm{~s}^{2}=$ & 16,05 & $\mathrm{~s}=$ & 4,01 & \\
\hline
\end{tabular}

15 Schreibung mit Akzent übernommen nach Kubik 2001:23. 


\begin{tabular}{lrrrrrr|r}
$\begin{array}{l}\text { Temperatur mit Inter- } \\
\text { vall: }\end{array}$ & $\mathbf{2 0 0 , 0 0}$ Cents & (Hexatonik) & \\
Teiltöne (Hz) & 325,00 & 364,80 & 409,47 & 459,62 & 515,91 & 579,08 & 289,54 \\
Teiltöne (Cents) & 0,00 & 200,00 & 400,00 & 600,00 & 800,00 & 1000,00 & 1000,00 \\
Differenz (Hz) & 0,00 & 5,20 & 3,53 & 3,38 & $-11,91$ & 27,92 & $-1,54$ \\
Differenz (Cents) & 0,00 & 24,50 & 14,84 & 12,69 & $-40,42$ & 81,51 & $-9,25$ \\
Statistik (Cents) & $\mathrm{Mw}=$ & 216,30 & $\mathrm{~s}^{2}=$ & 3410,23 & $\mathrm{~s}=$ & 58,40 &
\end{tabular}

\section{$-74-$}

Bordunton ist eindeutig Platte 3, der 7. Teilton einer angenommenen harmonischen Reihe mit Grundton bei 46,43 Hz. Permanente Sekundklänge entstehen vor allem zwischen den Platten 3 und 2. Ein besonderes Rätsel gibt Platte 7 auf. Sie kommt im Stück nicht vor. Doch vor dem Beginn des Stücks beginnen die Jungen kurz ein anderes, das sie nach wenigen Sekunden wieder abbrechen. Darin fungiert Platte 2, also der hypothetische 8. Teilton, als Bordunton und auch Platte 7 wird verwendet, wie eindeutig aus dem Spektrogramm abzulesen ist. Doch wie erklärt sich die Stimmung? Nach der Hypothese einer harmonischen Reihe — die bei diesem Instrument außergewöhnlich gut passt und innerhalb der ungefähren Toleranzspanne liegt - kann Platte 7 nur den 6. (ist gleich 3.) Teilton repräsentieren, was Kubiks These (7.-13. bzw. 9.-15. Teilton, besonders der 3. Partialton würde vermieden) entschieden widerspricht. Dies hieße, dass sich die Skala nicht (!) in den verschiedenen Oktaven wiederholt. Die Abweichungen wären jedoch mit 57,63 Cents außergewöhnlich hoch. Der Rahmen der Oktave könnte dagegen gewahrt werden, indem man eine Oktavierung von $2 * 288$ ist gleich $576 \mathrm{~Hz}$ annimmt, was ungefähr den $607 \mathrm{~Hz}$ der Platte 1 entspricht. Dies würde allerdings bedeuten, dass die Oktave um $33 \mathrm{~Hz}$ gespreizt wäre, was wiederum in dieser Lage 90,75 Cents (!) entspricht.

\section{$-75-$}

Leichter fällt die Erklärung der merkwürdigen Platte 7 mit dem Konzept einer äquidistanten Hexatonik. Die Abweichungen liegen dann innerhalb der Toleranzspanne. Doch wie beim Instrument von Venijwa und Jenja schlägt ein Ton, hier Platte 1, massiv aus der Reihe mit 81,51 Cents Abweichung. Wiederum müssen Platte 1 und 7 als unwahrscheinlich extrem gespreizte Oktave gelten, die Abweichungen sind angesichts der äquidistanten Stimmung nur wenig wahrscheinlicher.

$$
-76-
$$

Will man verschiedene Klassen von Intervallen annehmen, müssten diese ca. 140, 190 und 225 Cents betragen. Die Abweichungen sind größer als bei dem Instrument von Venjiwa und Jenja, bleiben aber unter 20 Cents. 
Aus dem Gebiet entlang des Flusslaufs des Ruvuma liegen drei Aufnahmen aus Kubiks Forschungsreise von 1962 vor. Am 29. Oktober nahm er im Dorf Namaua (20 km von Mueda) eine 6-stäbige dimbila auf. Die Bauart war von jener der mangwilo-Xylophone im Gebiet der Mitukwe-Berge etwas verschieden: Zwischen den Bananenstämmen und den Xylophonplatten lagen Grasbüschelauflagen, an einem Ende waren die Platten nicht mit Trennstäbchen voneinander gesondert, sondern durchstochen. Es spielten Sambaleu Natila (Spieler II) und Alfonso José (Spieler I), beide ca. 20 Jahre alt und vom Stamm der Wamakonde. Spieler II wurde kujalula, Spieler I kutunduvanga genannt. Die Aufnahme beinhaltet das Xylophonstïck Nambili sowie die Skala des Instruments.

Tab. 3: Stimmung der dimbila von A. José und S. Natila (B 9029)

Bordunton/Referenzton: $425,00 \mathrm{~Hz}$

\begin{tabular}{|c|c|c|c|c|c|c|}
\hline Platten & 6 & 5 & 4 & 3 & 2 & 1 \\
\hline Sin.-Vgl. (Hz) & 424,00 & 457,00 & 511,00 & 543,00 & 592,00 & 652,00 \\
\hline S_Tools $(\mathbf{H z})$ & 425,00 & 458,00 & 511,00 & 550,00 & 588,00 & 657,00 \\
\hline Cents & 0,00 & 129,46 & 319,03 & 446,36 & 562,02 & 754,12 \\
\hline Intervalle $(\mathrm{Hz})$ & 33,00 & 53,00 & 39,00 & 38,00 & 69,00 & \\
\hline Intervalle (Cents) & 129,46 & 189,57 & 127,33 & 115,66 & 192,09 & \\
\hline \multicolumn{2}{|c|}{$\begin{array}{l}\text { OT-Reihe mit Grund- } \\
\text { ton: }\end{array}$} & 38,64 & $\mathbf{H z}$ & $=425 / 11$ & & \\
\hline Teiltöne (Nr.) & 11,00 & 12,00 & 13,00 & 14,00 & 15,00 & 17,00 \\
\hline Teiltöne (Hz) & 425,00 & 463,64 & 502,27 & 540,91 & 579,55 & 656,82 \\
\hline Teiltön & 0,00 & 150,64 & 289,21 & 417,51 & 536,95 & 753,64 \\
\hline Differer & 0,00 & $-5,64$ & 8,73 & 9,09 & 8,45 & 0,18 \\
\hline Differe & 0,00 & $-21,18$ & 29,82 & 28,85 & 25,07 & 0,48 \\
\hline Statistik $(\mathrm{Hz})$ & $\mathrm{Mw}=$ & 38,67 & $s^{2}=$ & 50,09 & $s=$ & 7,08 \\
\hline \multicolumn{2}{|c|}{$\begin{array}{l}\text { Temperatur mit Inter- } \\
\text { vall: }\end{array}$} & 150,00 & Cents & (Oktaton & & \\
\hline Teiltöne (Hz) & 425,00 & 463,47 & 505,41 & 551,16 & 601,04 & 655,44 \\
\hline Teiltöne (Cents) & 0,00 & 150,00 & 300,00 & 450,00 & 600,00 & 750,00 \\
\hline Differenz $(\mathrm{Hz})$ & 0,00 & $-5,47$ & 5,59 & $-1,16$ & $-13,04$ & 1,56 \\
\hline Differenz (Cents) & 0,00 & $-20,54$ & 19,03 & $-3,64$ & $-37,98$ & 4,12 \\
\hline Statistik (Cents) & $\mathrm{Mw}=$ & 150,82 & $\mathrm{~s}^{2}=$ & 1088,83 & $\mathrm{~s}=$ & 33,00 \\
\hline
\end{tabular}

$$
-78-
$$

Die Tonhöhenempfindung ist bei Platten 1-3 etwas unklar, da die Spitzen breitere Frequenzregionen umfassen: bei Platte $1654-660 \mathrm{~Hz}$, bei Platte 2 585-591 Hz und bei Platte 3 549-551 Hz. In die Tabelle wurden Mittelwerte aufgenommen. Die Klänge der Platten sind im Vergleich zu den mangwiloXylophonen wenig energiereich sowohl bei der Aufnahme des Stücks als auch bei der Aufnahme der Skala. 
Als Bordunton dient Platte 6, Sekunden entstehen vor allem zwischen den Platten 6 und 5. Die Skala des Instruments jedoch ist in mehrfacher Hinsicht außergewöhnlich. Zunächst scheinen die Abweichungen zur harmonischen Reihe mit Grundton bei 38,64 Hz nicht allzu groß zu sein, wenngleich die Unterschiedsschwelle leicht überschritten ist. Nicht weiter verwunderlich ist, dass gegen Kubiks Hypothesen die Teiltöne 12 und 14 verwendet werden, die ja nichts anderes als Oktavierungen der Teiltöne 3 bzw. 6 und 7 sind. Beide waren - unter Voraussetzung der Annahme einer Obertonstimmung - auch beim Instrument von Dixon, Rosário und Carlindo vertreten. Kaum glaublich hingegen ist, dass hier der 17. Teilton, noch dazu ohne größere Abweichung, genützt wird.

$$
-80-
$$

Nicht weniger erstaunt das Ergebnis unter der Annahme einer temperierten Stimmung. In diesem Fall müsste man eine ebenfalls sehr unwahrscheinliche (weil m. W. kaum in einem Tonsystem vorkommende) äquidistante achtstufige Skala, quasi eine temperierte Oktatonik, annehmen. Die Toleranzspanne allerdings würde nicht überschritten. Pointiert formuliert, bietet das Instrument unter diesen beiden Gesichtspunkten sehr ungewöhnliche Stimmungen; diese aber recht genau. In jedem Fall dürfte, wie auch immer man die Skala auf eine Oktave ergänzen will, keine sechstönige Leiter vorliegen. Als Standardintervalle könnten mit der üblichen Exaktheit ca. 120 und 190 Cents angenommen werden.

$$
-81-
$$

Am selben Tag, dem 29. Oktober 1962, nahm Kubik in Nachomba (30 km südöstlich von Moçimboa do Rovuma) wiederum eine 6-stäbige dimbila auf. Die Protokolle liefern keine eindeutigen Angaben, ob dieses Instrument wie die dimbila von José und Natila und jene aus Mitande oder wie die mangwilo-Xylophone aus der Mitukwe-Region konstruiert ist. Die Spieler waren zwei Burschen im Alter von ca. 18-20 Jahren vom Stamm der Wamakonde. Jeder Musiker spielte auf jeweils drei der sechs Tasten. Die Aufnahme besteht aus dem Xylophonstück Nambili (das nicht mit dem Stück B 9029 identisch ist) und der Skala des Xylophons. 
Tab. 4: Stimmung einer dimbila aus Nachomba (B 9038)

\section{Bordunton/Referenzton: $585,00 \mathrm{~Hz}$}

\begin{tabular}{|c|c|c|c|c|c|c|}
\hline Platten & 1 & 5 & 3 & 4 & 2 & 6 \\
\hline Sin.-Vgl. (Hz) & 583,00 & 648,00 & 696,00 & 770,00 & 850,00 & 948,00 \\
\hline S_Tools $(\mathrm{Hz})$ & 585,00 & 645,00 & 697,00 & 771,00 & 845,00 & 947,00 \\
\hline Cents & 0,00 & 169,04 & 303,27 & 477,95 & 636,62 & 833,91 \\
\hline $\mathrm{e}(\mathrm{Hz})$ & 60,00 & 52,00 & 74,00 & 74,00 & 102,00 & \\
\hline Intervalle (Cents) & 169,04 & 134,23 & 174,69 & 158,66 & 197,30 & \\
\hline \multicolumn{2}{|c|}{ OT-Reihe mit Grundton: } & $\mathbf{5 8 , 5 0}$ & $\mathbf{H z}$ & \multicolumn{2}{|c|}{$(=585 / 10)$} & \\
\hline Teiltöne (Nr.) & 10,00 & 11,00 & 12,00 & 13,00 & 14,00 & 16,00 \\
\hline Teiltöne & 585,00 & 643,50 & 702,00 & 760,50 & 819,00 & 936,00 \\
\hline Teilti & 0,00 & 165,00 & 315,64 & 454,21 & 582,51 & 813,69 \\
\hline Diffe & 0,00 & 1,50 & $-5,00$ & 10,50 & 26,00 & 11,00 \\
\hline Eents) & 0,00 & 4,03 & $-12,37$ & 23,74 & 54,11 & 20,23 \\
\hline Statistik $(\mathrm{Hz})$ & $\mathrm{Mw}=$ & 60,33 & $\mathrm{~s}^{2}=$ & 105,04 & $s=$ & 10,25 \\
\hline \multicolumn{2}{|c|}{$\begin{array}{l}\text { Temperatur mit Inter- } \\
\text { vall: }\end{array}$} & 171,43 & Cents & \multicolumn{2}{|c|}{ (Heptatonik) } & \\
\hline Teiltöne (Hz) & 585,00 & 645,89 & 713,12 & 787,35 & 869,31 & 959,79 \\
\hline Teiltöne (Cents) & 0,00 & 171,43 & 342,86 & 514,29 & 685,71 & 857,14 \\
\hline Differenz $(\mathrm{Hz})$ & 0,00 & $-0,89$ & $-16,12$ & $-16,35$ & $-24,31$ & $-12,79$ \\
\hline Differenz (C & 0,00 & $-2,39$ & $-39,59$ & $-36,33$ & $-49,10$ & $-23,23$ \\
\hline Statistik (Cents) & $\mathrm{Mw}=$ & 166,78 & $s^{2}=$ & 423,82 & $\mathrm{~s}=$ & 20,59 \\
\hline
\end{tabular}

$-82-$

Einen Sonderfall stellt in physikalischer Hinsicht Platte 5 dar, da dort der 2. Teilton mit $1688 \mathrm{~Hz}$ energiereicher ist als der 1. Teilton mit $645 \mathrm{~Hz}$. Der Unterschied beträgt knapp $4 \mathrm{~dB}$. Trotzdem ist die (meine) Tonhöhenwahrnehmung eindeutig. Dies wohl deshalb, weil $645 \mathrm{~Hz}$ innerhalb des für die Tonhöhenwahrnehmung dominanten Bereiches liegt (siehe oben).

$$
-83-
$$

Bei dem Vergleich mit der harmonischen Reihe fällt eine relativ starke Abweichung bei Platte 2 auf, die Teiltöne 12 (3 bzw. 6) und 14 (7) sind erneut vertreten, der 16. Partialton entspricht dem vierfach oktavierten Grundton mit 58,5 Hz. Demgegenüber sind die Abweichungen beim Vergleich mit einer temperierten Stimmung eher gering und innerhalb der tolerablen Spanne. Allerdings muss erneut eine andere temperierte Reihe angenommen werden, nämlich eine äquidistante Heptatonik. Diese erscheint im betreffenden Kulturraum wesentlich wahrscheinlicher als eine temperierte Oktatonik oder Hexatonik, zumal sie in mehreren Musikkulturen Afrikas begegnet, nicht zuletzt auch im Süden Malawis (van Zanten 1980). Wiederum muss davon ausgegangen werden, dass bei einer Erweiterung der Skala mehr als sechs Skalentöne in den Oktavrahmen fallen. Standardintervalle lassen sich kaum feststellen, da die Intervalle nur geringe Unterschiede zueinander aufweisen. 
Auffällig ist indes, dass das gespielte Stück keinen eindeutig feststellbaren Bordun hat. Dies liegt wohl nicht zuletzt daran, dass aufgrund der Zuteilung von jeweils drei Platten zu einem Spieler kein Interlocking entstehen kann. Häufig geschlagen werden die Platten 1 und 5: die beiden tiefsten Tasten des Instruments, zwischen denen auch Sekundzusammenklänge entstehen. Hörbar sind diese jedoch kaum, da die Platte 1 äußerst leise ist. Ob dies am Instrument, an den Spielern oder an der Aufnahme liegt, konnte ich nicht ermitteln. Dazu kommt, dass die eindringlichste Platte eigentlich die höchste Platte 6 ist. Sie wird ebenfalls relativ häufig geschlagen und hat zumindest bei dieser Aufnahme einen wesentlich energiereicheren Klang als die Platten 1 und 5.

$$
-85-
$$

Kurzum: Sowohl das Stück als auch die Skala weichen nicht unerheblich vom Schema des einleitend beschriebenen Xylophonspiels ab, wie mir nicht zuletzt eine detaillierte Transkription des Stücks zeigte, die hier jedoch nicht Gegenstand der Untersuchung ist. Dabei fiel auf, dass die Spieler mitunter unregelmäßig schlagen, sowohl was Intensität als auch was den genauen Zeitpunkt des Schlages angeht. Die Irritationen ließen sich eventuell dadurch auflösen, dass sich hier zwei wenig erfahrene Spieler mit Xylophonspiel die Zeit vertreiben.

$$
-86-
$$

Einen Tag später als die letzten beiden Aufnahmen, am 30. Oktober 1962, machte Kubik Aufnahmen in Mitande (ca. $25 \mathrm{~km}$ östlich von Moçimboa do Rovuma). Dort spielten zwei ca. 16-17 Jahre alte Jungen vom Stamm der Wamakonde auf einer 8-stäbigen dimbila, wobei jedoch immer nur auf sechs Platten gespielt wurde. Das Instrument hatte wie jenes von José und Natila Grasbüschelauflagen, die Tasten waren an einem Ende zwecks Befestigung durchstochen. Die Aufnahme umfasst ein Xylophonstück sowie die Skala jener sechs Platten, auf denen gespielt wurde. 
Tab. 5: Stimmung einer dimbila aus Mitande (B 9145)

\section{Bordunton/Referenzton: $531,00 \mathrm{~Hz}$}

\begin{tabular}{|c|c|c|c|c|c|c|}
\hline Platten & 6 & 4 & 5 & 3 & 2 & 1 \\
\hline Sin.-Vgl. (Hz) & 478,00 & 532,00 & 589,00 & 645,00 & 709,00 & 787,00 \\
\hline S_Tools (Hz) & 481,00 & 531,00 & 583,00 & 645,00 & 712,00 & 787,00 \\
\hline Cents & 1028,79 & 0,00 & 161,74 & 336,70 & 507,80 & 681,18 \\
\hline Intervalle $(\mathrm{Hz})$ & 50,00 & 52,00 & 62,00 & 67,00 & 75,00 & \\
\hline Intervalle (Cents) & 171,21 & 161,74 & 174,96 & 171,09 & 173,38 & \\
\hline \multicolumn{2}{|c|}{ OT-Reihe mit Grundton: } & 59,00 & $\mathbf{H z}$ & $(=531 / 9$ & & \\
\hline Teiltöne & 8,00 & 9,00 & 10,00 & 11,00 & 12,00 & 13,00 \\
\hline Teiltöne (Hz) & 472,00 & 531,00 & 590,00 & 649,00 & 708,00 & 767,00 \\
\hline Teiltöne (Cents) & 996,09 & 0,00 & 182,40 & 347,41 & 498,04 & 636,62 \\
\hline Differenz $(\mathrm{Hz})$ & 9,00 & 0,00 & $-7,00$ & $-4,00$ & 4,00 & 20,00 \\
\hline Differenz (Cents) & 32,70 & 0,00 & $-20,66$ & $-10,70$ & 9,75 & 44,56 \\
\hline Statistik $(\mathrm{Hz})$ & $\mathrm{Mw}=$ & 61,20 & $\mathrm{~s}^{2}=$ & 85,96 & $\mathrm{~s}=$ & 9,27 \\
\hline \multicolumn{2}{|c|}{$\begin{array}{l}\text { Temperatur mit Inter- } \\
\text { vall: }\end{array}$} & 171,43 & Cents & \multicolumn{2}{|c|}{ (Heptatonik) } & \\
\hline Teiltöne (Hz) & 480,94 & 531,00 & 586,27 & 647,30 & 714,67 & 789,06 \\
\hline Teiltöne (Cents) & 1028,57 & 0,00 & 171,43 & 342,86 & 514,29 & 685,71 \\
\hline Differenz (Hz) & 0,06 & 0,00 & $-3,27$ & $-2,30$ & $-2,67$ & $-2,06$ \\
\hline Differenz (Cents) & 0,22 & 0,00 & $-9,69$ & $-6,15$ & $-6,49$ & $-4,53$ \\
\hline Statistik (Cents) & $\mathrm{Mw}=$ & 170,48 & $s^{2}=$ & 20,16 & $s=$ & 4,49 \\
\hline
\end{tabular}

$-87-$

Bei der Aufnahme der Stimmung sind die Platten gegenüber der Aufnahme des Stücks sehr leise angeschlagen. Für Platte 6, die eigentlich unter dem Referenzton liegt, wurde der Übersichtlichkeit halber um 1200 Cents korrigiert.

$$
-88-
$$

Der Bordunton liegt auf Platte 4, die typischen Sekunden ergeben sich aus dem häufigen Zusammenklang der Platten 4 und 5. Die musikalische Struktur entspricht, soweit man aus der Aufnahme erkennen kann, dem Stil der Mitukwe-Region. Die Skala ist zuverlässiger bestimmbar als in allen bisherigen Fällen. Eine harmonische Reihe liegt mit großer Sicherheit nicht vor, die Abweichungen sind zu groß (32,7 und 44,56 Cents). Die Übereinstimmungen mit einer temperierten Heptatonik indes sind frappant. Die Abweichungen liegen - Zufall oder nicht? - weit unter jeder denkbaren Toleranzschwelle. Die einzige Intervallklasse beträgt demnach (gerundet) 171 Cents.

$$
-89-
$$

1983 machte Kubik weitere Aufnahmen vom Xylophonspiel der hier diskutierten Art. Am 18. Juli 1983 besuchte er in Makanjila (am Ostufer des Nyasa-Sees an der Grenze Malawi/Moçambique) den ca. 35 Jahre alten 
Schneider und Xylophonspieler Mdala Katema, der ein 8-züngiges mangolongondo mit verschiedenen anderen Musikern, darunter der junge Bursche Chibokozingwa (ca. 20 Jahre alt), spielte. (Mdala Katema spielte ansonsten mit seiner Frau, die jedoch bei diesen Aufnahmen nicht zugegen war.) Die Musiker gehörten zum Stamm der Ayao mit der Sprache Chiyao.

$$
-90-
$$

Jeder Musiker hält zwei Stäbchen, die milazi, was im Chichewa msungwi 'Bambus, Ried' entspricht. Beim Spiel war eine dritte Person beteiligt, ein Helfer, der die verschobenen Tasten wieder zurecht rückte. Diese Rolle wurde hier von James Amadi übernommen. Spieler I wurde kunchiji genannt, der Meister (Spieler II) - hier Mdala Katema - kundio, was den Chichewa/Chinyanja-Begriffen kumansere 'links' und kumanja 'rechts' entspricht. Die Stäbe des mangolongondo heißen lingolongondo. Sie können in ihrer Anordnung von Stück zu Stück vertauscht werden.

$$
-91-
$$

Von mehreren Aufnahmen Kubiks bei Mdala Katema wurden drei herangezogen, die ausschliesslich oder unter anderem die Skala des Instruments enthalten. B 28278 umfasst das Xylophonstück Anjembere sowie die Skala des Instruments, B 28281 das Xylophonstück Ankamulengangapezi chongwe ${ }^{16}$ und die Skala, B 28284 schliesslich die Stimmung des Xylophons mit Ansage und Stimmgabelton.

Tab. 6: Stimmung des mangolongondo von Mdala Katema (B 28284)

Bordunton/Referenzton: $303,00 \mathrm{~Hz}$

\begin{tabular}{lrrrrrrrr} 
Platten & \multicolumn{1}{c}{8} & \multicolumn{1}{c}{6} & \multicolumn{1}{c}{7} & \multicolumn{1}{c}{5} & \multicolumn{1}{c}{4} & \multicolumn{1}{c}{3} & \multicolumn{1}{c}{2} & \multicolumn{1}{c}{1} \\
\hline Sin.-Vgl. (Hz) & 254,00 & 300,00 & 337,00 & 363,00 & 399,00 & 433,00 & 483,00 & 558,00 \\
S_Tools (Hz) & $\mathbf{2 5 5 , 0 0}$ & $\mathbf{3 0 3 , 0 0}$ & $\mathbf{3 3 9 , 0 0}$ & $\mathbf{3 6 0 , 0 0}$ & $\mathbf{4 0 2 , 0 0}$ & $\mathbf{4 3 5 , 0 0}$ & $\mathbf{4 8 2 , 0 0}$ & $\mathbf{5 6 2 , 0 0}$ \\
Cents & 901,42 & 0,00 & 194,36 & 298,41 & 489,45 & 626,04 & 803,66 & 1069,50 \\
\hline Intervalle (Hz) & 48,00 & 36,00 & 21,00 & 42,00 & 33,00 & 47,00 & 80,00 & \\
Intervalle (Cents) & 298,58 & 194,36 & 104,05 & 191,04 & 136,58 & 177,62 & 265,84 & \\
\hline OT-Reihe mit Grund- & $\mathbf{3 7 , 8 8} \mathbf{H z}$ & $=303 / 8$ & & & & \\
ton: & & & & & & & & \\
Teiltöne (Nr.) & 7,00 & 8,00 & 9,00 & 10,00 & 11,00 & 12,00 & 13,00 & 15,00 \\
Teiltöne (Hz) & 265,13 & 303,00 & 340,88 & 378,75 & 416,63 & 454,50 & 492,38 & 568,13 \\
Teiltöne (Cents) & 968,83 & 0,00 & 203,91 & 386,31 & 551,32 & 701,96 & 840,53 & 1088,27 \\
Differenz (Hz) & $-10,13$ & 0,00 & $-1,88$ & $-18,75$ & $-14,63$ & $-19,50$ & $-10,38$ & $-6,13$ \\
Differenz (Cents) & $-67,41$ & 0,00 & $-9,55$ & $-87,90$ & $-61,86$ & $-75,92$ & $-36,87$ & $-18,77$ \\
Statistik (Hz) & $\mathrm{Mw}=$ & 38,38 & $\mathrm{~s}^{2}=$ & 63,90 & $\mathrm{~s}=$ & 7,99 & &
\end{tabular}

16 Das Stück ist (nach Kubik 1983/84) identisch mit einem der 1962 in den MitukweBergen aufgenommenen Stücken. 
Der vor der Stimmung aufgenommene Stimmgabelton liegt bei $435 \mathrm{~Hz}$. Bei Platte 8 wurde der besseren Orientierung halber für die Centswerte eine Oktavbereinigung vorgenommen. Die Aufnahmen der Skala bei B 28278 und B 28281 weichen um wenige Cents von den Werten bei B 28284 ab. Der Grund muss in einer leichten Varianz der Bandgeschwindigkeit gelegen haben. Die in Tab. 6 gegebenen Werte wurden nach B 28284 ermittelt.

\section{- 93 -}

Die beiden Stücke entsprechen weitgehend den bekannten Strukturschemata. Lediglich die Frage, welcher Ton als Bordunton fungiert, ist für die Aufnahme B 28278 nicht eindeutig zu beantworten. Es könnte sowohl Platte 6 als auch Platte 8 sein. Die Häufigkeit des Anschlagens dieser Platten ist ungefähr gleich, energiereicher ist Platte 6. Dies nicht zuletzt deshalb, weil Platte 8 die stärkste tonale Komponente bei $759 \mathrm{~Hz}$ hat. Der Bereich um $252 \mathrm{~Hz}$ liegt um beinahe $10 \mathrm{~dB}$ unter dieser Spitze. Dies hat zur Folge, dass keine der beiden Tonhöhen klar zu hören ist, der Tonhöheneindruck ständig umschlägt und im Kontext der übrigen Platten im Xylophonstück weitgehend untergeht. Eindeutigkeit dagegen liegt vor für B 28281. Hier übernimmt Platte 6 die Funktion des Borduns. Auch bei dieser Platte ist der 2. Teilton mit $855 \mathrm{~Hz}$ um ca. $5 \mathrm{~dB}$ stärker als die untere Klangkomponente. Als Grundton wird jedoch - wohl wegen der Erwartungshaltung aus dem Umfeld der übrigen, eher tiefen Xylophonplatten — recht eindeutig die Tonhöhe bei $300 \mathrm{~Hz}$ empfunden. Die charakteristischen Sekunden ergeben sich in beiden Stücken aus dem Zusammenklang von Platte 8 und 6 .

$$
-94-
$$

Als Deutungsmuster für die Skala des Instruments bieten sich hier nur die Obertonreihe und die Annahme von Intervallklassen an. Ein Vergleich mit einer temperierten Stimmung wäre unsinnig, wie die Cents-Intervalle zwischen den Tasten deutlich vorführen. Man müsste mit Abweichungen von über 100 Cents rechnen. Allerdings sind auch die Abweichungen von einer idealen harmonischen Reihe mit Grundton $37,88 \mathrm{~Hz}$, die erneut den 12. (ist gleich 6. ist gleich 3.) Teilton enthielte, zu groß, als dass die Skala damit zu erklären wäre. Am ehesten wird man die Stimmung als Reihung von Intervallen mit Schritten von ca. 125 bzw. 190 Cents auflösen können. Zwischen den Platten 8 und 6 sowie 2 und 1 liegen Doppelintervalle vor. Die Intervalle bewegen sich ungefähr innerhalb der angenommenen Toleranzspanne.

$$
-95-
$$

Bemerkenswert an der Stimmung dieses Instruments ist, dass offenbar das Konzept des Oktavrahmens nicht genützt wird, da in diesem Fall eine Wiederholung des 7. Partialtons als 14. Teilton zu erwarten wäre. Ähnliches deutete sich bei der Untersuchung des Xylophons von Dixon, Rosário und 
Carlindo an. Die Kritik an den Centsangaben Kubiks (siehe oben) muss demnach zurückgenommen werden. Zwar ist nach wie vor eine negative Cents-Zahl ungewöhnlich - doch dies allein deshalb, weil man so sehr an das Konzept der Oktave gewöhnt ist. Ein methodologischer Widerspruch zu den Skalen scheint indes nicht vorzuliegen.

$$
-96-
$$

Den Höhepunkt der Reihe von Aufnahmen zum Xylophonspiel des Nyasa/Ruvuma-Gebiets bilden zweifellos jene Stücke, die Kubik bei Waisoni Msusa, einem ca. 35 Jahre alten Xylophonvirtuosen vom Stamm der Ayao (Sprache Chiyao) machen konnte. Kubik beteuerte selbst mehrmals die hervorragende Virtuosität dieses Spielers (siehe beispielsweise Kubik 1983/84; 1987:43). Um Waisoni Msusa hatte sich in Nkopiti, einem Teil des Dorfes Lukono (ca. $5 \mathrm{~km}$ nördlich von Makanjila), eine regelrechte mangolongon$d o$-Schule gebildet; ein Kreis von mehr als zehn Personen, aus dem wiederum besonders der sehr talentierte Junge Tawina Ndala, ca. 8 Jahre alt, auffiel. Die hier ausgewerteten Aufnahmen datieren auf den 22. Juli 1983.

$$
-97-
$$

Die Bezeichnungen für die einzelnen Strukturelemente des Xylophonspiels weichen - trotz der geringen Entfernung zu Mdala Katema! — von den bisher erwähnten ab. Die Schlägel heißen yikongo (Pl., Sg.: chikongo), die Musiker werden wakwimba 'der Singende' (Spieler II) und wakwitichisya 'der Antwortende' (Spieler I) genannt. ${ }^{17}$ Gespielt wird auf einem 9-züngigen mangolongondo, das auf zwei Arten geschlagen wird: bei 'klingenden' Schlägen wird die Platte einfach angeschlagen, beim 'Stoppschlag' wird der Schlägel verzögert hochgehoben und die Platte entsprechend gedämpft.

Für die Untersuchung herangezogen wurden insgesamt vier Aufnahmen, von denen drei Xylophonstücke beinhalten und die vierte die Stimmung des Instruments dokumentiert. Die Stücke sind auch auf der von Kubik publizierten Doppel-LP (Kubik 1989; danach die Schreibung und Übersetzung der Titel) auf Seite C, Nr. 1-3 zugänglich. Es handelt sich um das Xylophonstück Ulendo wa kumigunda 'Die Reise zu den Feldern' (B 28328), gespielt von Waisoni Msusa und einer weiteren Person (Transkription bei Kubik 1987:38-41; 1989:43), das Xylophonstück Kung'anda kwa anyamata 'Das Spiel für Jungen’ (B 28336), gespielt von Dengamu Mponda (ca. 17 Jahre alt) und Tawina Ndala, und das Xylophonstück Maguluve kumala imanga kumigunda 'Die Wildschweine fressen den Mais auf den Feldern auf' (B

17 Schreibung und Übersetzung nach Kubik 1987:34. In Protokollen (Kubik 1983/84) stets Schreibungen mit anlautenden <v>. Inkonsequent: „vakwiticisya“ (Kubik 1987:38; Hervorhebung original). 
28343), gespielt von Waisoni Msusa, Tawina Ndala und Dengamu Mponda. Die Stimmung des mangolongondo inklusive Ansage und Stimmgabelton gibt B 28330, die neun Platten werden nach Angabe von links nach rechts aus der Sicht des Experten angeschlagen. Tatsächlich hieße dies, dass die Platten in der exakt entgegengesetzten Reihenfolge liegen als bei den von Kubik (1987:39; 1989:10) gegebenen Skalen für die Stücke B 28328 und B 28343. Dort ist die Reihenfolge zu jener bei B 28330 (siehe die Nummerierung der Platten in Tab. 7) umgekehrt. Eher wird hier eine Rechts-LinksVerwechslung vorliegen: die Platten werden aus der Sicht des Experten von rechts nach links angeschlagen. Messungen der Stimmung durch Kubik mit einem Korg Chromatic Tuner WT-12 erbrachten jedenfalls folgende Ergebnisse (Kubik 1987:39; 1989:10): 550 (1201) - 503 (1045) - 447,5 (843) 394 (623) - 350,5 (421) - 314,5 (233) - 275 (0) - 243,5 (988) - 618 (1403) $\mathrm{Hz}$ (Cents).

Tab. 7: Stimmung des mangolongondo von Waisoni Msusa (B 28330)

\begin{tabular}{|c|c|c|c|c|c|c|c|c|c|}
\hline $\begin{array}{l}\text { Bordunton/ } \\
\text { Referenzton: }\end{array}$ & & 271,00 & $\mathbf{H z}$ & & & & & & \\
\hline Platten & 2 & 3 & 4 & 5 & 6 & 7 & 8 & 9 & 1 \\
\hline $\begin{array}{l}\text { Kubik 1987:39 } \\
(\mathrm{Hz})\end{array}$ & 243,50 & 275,00 & 314,50 & 350,50 & 394,00 & 447,50 & 503,00 & 550,00 & 618,00 \\
\hline $\begin{array}{l}\text { Diff. Zu S_T. } \\
\text { (Ct.) }\end{array}$ & 25,06 & 25,37 & 24,95 & 22,37 & 22,11 & 25,33 & 20,78 & 18,99 & 25,40 \\
\hline Statistik (Cents) & $\mathrm{Mw}=$ & 23,37 & $\mathrm{~s}^{2}=$ & 4,09 & $\mathrm{~s}=$ & 2,02 & & & \\
\hline $\begin{array}{l}\text { Kubik, korr. } \\
(\mathrm{Hz})\end{array}$ & 240,23 & 271,31 & 310,28 & 345,80 & 388,72 & 441,50 & 496,25 & 542,62 & 609,71 \\
\hline Sin.-Vgl. (Hz) & 243,00 & 273,00 & 309,00 & 347,00 & 390,00 & 439,00 & 500,00 & 544,00 & 607,00 \\
\hline S_Tools $(\mathrm{Hz})$ & 240,00 & 271,00 & 310,00 & 346,00 & 389,00 & 441,00 & 497,00 & 544,00 & 609,00 \\
\hline Cents & 989,69 & 0,00 & 232,77 & 422,98 & 625,77 & 842,98 & 1049,94 & 1206,38 & 1401,78 \\
\hline Intervalle $(\mathrm{Hz})$ & 31,00 & 39,00 & 36,00 & 43,00 & 52,00 & 56,00 & 47,00 & 65,00 & \\
\hline $\begin{array}{l}\text { Intervalle } \\
\text { (Cents) }\end{array}$ & 210,31 & 232,77 & 190,20 & 202,80 & 217,21 & 206,96 & 156,43 & 195,40 & \\
\hline $\begin{array}{l}\text { Temperatur } \\
\text { mit Intervall: }\end{array}$ & & 200,00 & Cents & $\begin{array}{r}\text { (Hexa- } \\
\text { tonik) }\end{array}$ & & & & & \\
\hline Teiltöne (Hz) & 241,43 & 271,00 & 304,19 & 341,44 & 383,25 & 430,19 & 482,87 & 542,00 & 608,37 \\
\hline Teiltöne (Cents) & 1000,00 & 0,00 & 200,00 & 400,00 & 600,00 & 800,00 & 1000,00 & 1200,00 & 1400,00 \\
\hline Differenz (Hz) & $-1,43$ & 0,00 & 5,81 & 4,56 & 5,75 & 10,81 & 14,13 & 2,00 & 0,63 \\
\hline $\begin{array}{l}\text { Differenz } \\
\text { (Cents) }\end{array}$ & $-10,31$ & 0,00 & 32,77 & 22,98 & 25,77 & 42,98 & 49,94 & 6,38 & 1,78 \\
\hline Statistik (Cents) & $\mathrm{Mw}=$ & 201,51 & $\mathrm{~s}^{2}=$ & 440,20 & $\mathrm{~s}=$ & 20,98 & & & \\
\hline
\end{tabular}

Der Ton der Stimmgabel lag bei $434 \mathrm{~Hz}$. Die Cents-Werte von Platte 2 wurden der Lesbarkeit halber oktavbereinigt. Die Werte aus den Messungen Kubiks sind um ca. 23 Cents korrigiert, da offensichtlich entweder das Messgerät falsch kalibriert war oder eine andere Bandgeschwindigkeit vor- 
lag. Dies ist freilich nicht von Bedeutung, da die relativen Tonhöhenverhältnisse davon nicht beeinträchtigt sind.

$$
-100-
$$

Allgemein ist zu bemerken, dass die Platten äußerst klangreich sind und durch lange Tondauern auffallen. Der zweite Teilton ist relativ lange präsent bei den Platten 2-4, was zu mehrdeutigen Tonhöhenempfindungen führt. Neben die tiefen Spitzen bei 240, 271 und $310 \mathrm{~Hz}$ treten solche mit (in dieser Reihung) 862, 953 und $1041 \mathrm{~Hz}$. Im Falle der Taste 2 ist die zweite Spitze um ca. 4 dB energiereicher als die erste. Ansonsten dürfte die Auffälligkeit dieser hohen Spitzen in ihrer Lage bzw. in ihrem für die Wahrnehmung von Tonhöhen dominanten Bereich begründet sein. Die übrigen Platten verhalten sich wie gewohnt: die höheren Frequenzbereiche verlieren rasch an Energie, während ein einzelner Teilton die tonalen Charakteristika des Klangs bestimmt.

$$
-101-
$$

Die Struktur entspricht dem oben vorgestellten Schema. Alle drei Stücke verwenden einen Bordun, der stets bei $271 \mathrm{~Hz}$ auf Platte 3 liegt. Bei B 28343 wird der Bordunton (bei dieser Aufnahme sehr leise; Position des Mikrofons?) zusätzlich betont, indem einer der Anwesenden mit einer Männerstimme gelegentlich eine Formel singt, die immer wieder knapp über dem Bordunton ansetzt und durch eine fallende Stimmbewegung auf diesem zu ruhen kommt. Also kann davon ausgegangen werden, dass man sich der Bedeutung dieser zentralen Tonstufe auch in den betreffenden Musikkulturen bewusst ist und dass hier nicht nur Konzepte aus anderen Regionen auf die betreffende Musikkultur projiziert werden.

\section{$-102-$}

Ähnliches gilt für das Prinzip der Sekundzusammenklänge. Wieder beim Stück B 28343 - doch nicht nur bei diesem (siehe Kubik 1983/84) spielen drei Personen aktiv am Instrument. Einander gegenüber sitzen der wakwitichisya, hier Waisoni Msusa, und der wakwimba, hier Tawina Ndala. Letzterer spielt nur auf den mittleren sieben Tasten, während Waisoni Msusa auch auf der (aus seiner Sicht) äußersten linken Platte spielt, die bei diesem Stück die Platte 9 mit $544 \mathrm{~Hz}$ ist. Platte $1(609 \mathrm{~Hz})$ dagegen, am anderen Ende des Instruments gelegen, wird ausschließlich vom dritten Spieler, hier Dengamu Mponda, geschlagen, dessen Spielbereich nur aus dieser Platte besteht (siehe die schematische Darstellung bei Kubik 1989:10, Abb. 4). Waisoni Msusa spielt nun auf Platte 9 die zwölf-tönige Time-LineFormel [x.x.x..x.x..], die parallel von Dengamu Mponda auf Platte 1 geschlagen wird. Sie erklingt also in parallelen Sekunden im Abstand von ca. 195 Cents (nach Kubik 1989:10). 
Die Notizen sollten bereits gezeigt haben, mit welcher Professionalität hier das mangolongondo-Spiel praktiziert und auch an jüngere Schüler vermittelt wird (dazu Kubik 1987:33-34, 38-46; 1989: 10-11, 42-43). Wie aber ist die Stimmung dieses besonderen Xylophons beschaffen? Ein Vergleich mit der harmonischen Reihe wird hier, wie die Hertz-Zahlen der Platten deutlich vorführen, zu keinem rechten Ergebnis führen. Die verschiedenen Intervalle zwischen 31 und $65 \mathrm{~Hz}$ mit relativ gleichmäßig verteilten Zwischenstufen lassen sich keinen Gruppen nach dem Muster x, $2 * x \mathrm{~Hz}$ usf. zuordnen. Der Vergleich wäre ebenso wenig sinnvoll wie jener des mangolongondo von Mdala Katema mit einer temperierten Reihe.

\section{$-104-$}

Relativ gut fügt sich die Skala indes in das Konzept einer temperierten Hexatonik ein. Die Abweichungen bleiben innerhalb der postulierten Toleranzspanne. Für diese Interpretation spricht ferner das Konzept des Oktavrahmens, wie er hier besonders bei der Oktavierung des Borduntons angewendet wird und - mit größeren Abweichungen - auch bei den Platten 2 : 8 und $4: 1 \mathrm{zu}$ beobachten ist. Dafür, dass die Musiker die Oktavierungen besonders des Borduntons absichtlich vornehmen, spricht die in parallelen Sekunden gespielte Time-Line-Formel, die so gesehen wiederum die Bedeutung des Borduntons herausstreicht.

\section{$-105-$}

Am selben Tag wurde an einem anderen Xylophon, das nur wenige hundert Meter vom Xylophon Waisoni Msusas im Schatten stand und dessen Eigentümer beim Fußballspiel war, die Stimmung (wiederum inklusive Stimmgabelton) abgenommen. Das Xylophon war von Jungen gebaut worden. 
Tab. 8: Stimmung eines mangolongondo aus Lukono (B 28347)

\begin{tabular}{|c|c|c|c|c|c|c|c|c|c|}
\hline $\begin{array}{l}\text { Bordunton/ } \\
\text { Referenzton: }\end{array}$ & & 217,00 & $\mathrm{~Hz}$ & & & & & & \\
\hline Platten & 5 & 9 & 8 & 7 & 6 & 4 & 3 & 2 & 1 \\
\hline Sin.-Vgl. (Hz) & 215,00 & 258,00 & 279,00 & 299,00 & 315,00 & 334,00 & 371,00 & 398,00 & 422,00 \\
\hline S_Tools $(\mathrm{Hz})$ & 217,00 & 255,00 & 280,00 & 300,00 & 314,00 & 333,00 & 368,00 & 398,00 & 421,00 \\
\hline Cents & 0,00 & 279,36 & 441,28 & 560,72 & 639,68 & 741,39 & 914,41 & 1050,09 & 1147,35 \\
\hline Intervalle $(\mathrm{Hz})$ & 38,00 & 25,00 & 20,00 & 14,00 & 19,00 & 35,00 & 30,00 & 23,00 & \\
\hline $\begin{array}{l}\text { Intervalle } \\
\text { (Cents) }\end{array}$ & 279,36 & 161,92 & 119,44 & 78,96 & 101,71 & 173,02 & 135,68 & 97,26 & \\
\hline $\begin{array}{l}\text { OT-Reihe mit } \\
\text { Grundton: }\end{array}$ & & 25,50 & $\mathrm{~Hz}$ & $(=\mathrm{Mw})$ & & & & & \\
\hline Teiltöne (Nr.) & 9,00 & 10,00 & 11,00 & 12,00 & 13,00 & 14,00 & 15,00 & 16,00 & 17,00 \\
\hline Teiltöne (Hz) & 229,50 & 255,00 & 280,50 & 306,00 & 331,50 & 357,00 & 382,50 & 408,00 & 433,50 \\
\hline Teiltöne (Cents) & 96,96 & 279,36 & 444,37 & 595,00 & 733,58 & 861,87 & 981,32 & 1093,05 & 1198,00 \\
\hline Differenz $(\mathrm{Hz})$ & $-12,50$ & 0,00 & $-0,50$ & $-6,00$ & $-17,50$ & $-24,00$ & $-14,50$ & $-10,00$ & $-12,50$ \\
\hline $\begin{array}{l}\text { Differenz } \\
\text { (Cents) }\end{array}$ & $-96,96$ & 0,00 & $-3,09$ & $-34,28$ & $-93,89$ & $-120,48$ & $-66,90$ & $-42,96$ & $-50,65$ \\
\hline Statistik $(\mathrm{Hz})$ & $\mathrm{Mw}=$ & 25,50 & $\mathrm{~s}^{2}=$ & 58,75 & $\mathrm{~s}=$ & 7,66 & & & \\
\hline $\begin{array}{l}\text { Temperatur } \\
\text { mit Intervall: }\end{array}$ & & 150,00 & Cents & $\begin{array}{r}\text { (Oktato- } \\
\text { nik) }\end{array}$ & & & & & \\
\hline Teiltöne (Hz) & 217,00 & 236,64 & 258,06 & 281,41 & 306,88 & 334,66 & 364,95 & 397,98 & 434,00 \\
\hline Teiltöne (Cents) & 0,00 & 150,00 & 300,00 & 450,00 & 600,00 & 750,00 & 900,00 & 1050,00 & 1200,00 \\
\hline Differenz (Hz) & 0,00 & 18,36 & 21,94 & 18,59 & 7,12 & $-1,66$ & 3,05 & 0,02 & $-13,00$ \\
\hline $\begin{array}{l}\text { Differenz } \\
\text { (Cents) }\end{array}$ & 0,00 & 129,36 & 141,28 & 110,72 & 39,68 & $-8,61$ & 14,41 & 0,09 & $-52,65$ \\
\hline Statistik (Cents) & $\mathrm{Mw}=$ & 143,42 & $\mathrm{~s}^{2}=$ & 3543,83 & $\mathrm{~s}=$ & 59,53 & & & \\
\hline
\end{tabular}

Die Stimmgabel lag bei $435 \mathrm{~Hz}$. Das Xylophon ist, ebenso wie das Instrument von Waisoni Msusa, sehr klangreich. Fast alle Platten zeigen einen relativ starken zweiten Teilton, der bei den Platten 5-9 eine ambivalente Tonhöhenempfindung bewirkt. Neben die erste tonale Komponente treten Teiltöne mit 642, 883, 806, 786 und $696 \mathrm{~Hz}$, wobei bei den Platten 5 und 8 die höheren Teiltöne um ca. 2 bzw. $5 \mathrm{~dB}$ energiereicher sind.

$$
-107-
$$

Die Skala ist mit keiner der drei bislang versuchten Hypothesen in Einklang zu bringen. Die Abweichungen sowohl zu einer harmonischen Reihe mit 25,5 Hz Grundton (der Mittelwert der Hz-Schritte) als auch zu einer temperierten Heptatonik überschreiten die angenommenen Toleranzspannen bei weitem. Ähnliches gilt für die Annahme von verschiedenen Standardintervallen. Die Intervalle sind zu verschieden, als dass sie einzelnen distinktiven Klassen beigeordnet werden könnten. Ich vermute, dass dieses Instrument noch nicht seine endgültige Form erreicht hat. Die einzelnen Platten sind entweder nur ungefähr oder gar nicht gestimmt. Vielleicht kann auch an die Methode des Stimmens gedacht werden, die Kubik bei Venjiwa und Jenja 
beobachten konnte (siehe oben): Aus einer großen Anzahl von Platten werden die ungefähr passenden ausgewählt und dann feingestimmt. Dieser zweite Teil des Stimmvorgangs, das Feinstimmen, ist für dieses Instrument wohl noch durchzuführen. Möglicherweise sind auch noch Platten auszuscheiden, bedenkt man die so ungewöhnlich kleinen Intervalle zwischen den Platten 7 und 6 sowie 2 und 1.

\section{$* * *$ \\ $-108-$}

Wie lassen sich die mitunter sehr disparaten Ergebnisse der einzelnen Untersuchungen an den Instrumenten zusammenfassen? Am ehesten wird man eine Teilung in drei Gruppen veranschlagen können, wobei das letzte Xylophon, dessen Stimmung sehr fragwürdig erscheint, ausgeklammert sei.

$$
-109-
$$

Unter eine erste Gruppe fasse ich die Aufnahmen B 9038 und B 9145: zwei Xylophone aus dem Ruvuma-Tal. Bereits die Struktur der gespielten Stücke weicht hier - zumindest bei B 9038 - entscheidend von der musikalischen Eigenheit der übrigen Stücke ab (kein Interlocking, kein Bordun, viele Unregelmäßigkeiten). Ähnliches kann für die Stimmung gelten. Denn entgegen allen anderen herangezogenen Instrumenten scheinen diese beiden dimbilaXylophone nach einer temperierten Heptatonik gestimmt. Bei B 9038 bleiben die Abweichungen im Rahmen der von Kubik für die Pentatonik Bugandas und von van Zanten für die Heptatonik im Süden Malawis implizierten Spanne, bei B 9145 liegen sie weit darunter. Jede andere versuchte Hypothese müsste mit weit größeren Toleranzintervallen rechnen. Ebenfalls für eine temperierte Heptatonik spricht die Tatsache, dass diese Stimmung generell nicht unüblich und beispielsweise auch im Süden Malawis verbreitet ist. Mit B 9038 und B 9145 ist folglich eine etwas abweichende Stilschicht erfasst, wenngleich die geographischen Unterschiede (zur B 9029) nur wenige Kilometer betragen. Eine Erklärung dafür könnte die dichte Bevölkerung besonders des unteren Ruvuma-Laufs, die Besiedlung durch verschiedenste ethnische Gruppen sein, was ein entsprechend vielfältiges kulturelles Spektrum bedingt.

$$
-110-
$$

Die zweite Gruppe besteht aus den mangwilo-Xylophonen von Venjiwa und Jenja (B 8995) sowie von Dixon, Rosário und Carlindo (B 9088), aus der dimbila von Sambaleu Natila und Alfonso José (B 9029) sowie aus dem mangolongondo von Mdala Katema (B 28284). Für alle vier Instrumente ist eine eindeutige Zuordnung zur Obertonreihe oder zur Temperatur nicht möglich. Das mangwilo von Venjiwa und Jenja scheint eher einer harmoni- 
schen Reihe als einer äquidistanten sechstönigen Stimmung zu entsprechen, doch darf nicht übersehen werden, dass bei der Folie einer harmonischen Reihe viel geringere Toleranzspannen zu veranschlagen wären. Letztlich passen also beide Hypothesen mehr schlecht als recht zur Skala des Xylophons. Gleiches gilt für das mangwilo von Dixon, Rosário und Carlindo. Dessen sehr stark gespreizte Oktave zwischen den Platten 1 und 7 führt die Aporie der beiden Hypothesen klar vor Augen: Platte 1 passt zur Obertonreihe, Platte 7 zur temperierten Skala.

Noch problematischer ist die Skala der dimbila von Alfonso José und Sambaleu Natila. Zwar erstaunt es wenig, dass - bei der Annahme einer harmonischen Reihe - die Teiltöne 12 und 14 (ist gleich 3 bzw. 6 und 7) genützt werden; eine Orientierung am 17. Teilton jedoch scheint aus praktischen Gründen völlig undenkbar. Ebenso ist eine temperierte achttönige Skala m. W. derart unüblich, dass auch sie als Erklärung dieser Skala ausscheidet, wenngleich insbesondere bei der Hypothese einer äquidistanten Stimmung die Abweichungen von der idealen Skala nicht allzu groß wären. Das mangolongondo von Mdala Katema schließlich passt zu keiner denkbaren temperierten Skala, auch die Abweichungen von einer harmonischen Reihe sind übermäßig stark. Weniger erstaunt, dass auch hier wiederum mit dem 7. (ist gleich 14.) und 12. (ist gleich 3. bzw. 6.) Teilton zu rechnen wäre.

$-112-$

Somit bleibt von drei erprobten Interpretationsmöglichkeiten nur eine übrig: die Annahme von Standardintervallen. Und diese passt überraschend gut zu allen vier Instrumenten. Es ergibt sich sogar, dass für alle vier Skalen dieselben drei (bei B 9029 und 28284 nur zwei) Arten von Intervallen als ideale Muster angenommen werden können. Die drei Standardintervalle mit 126 (A), 189 (B) und 216 (C) Cents geben gute Annäherungen, um die Abweichungen der tatsächlichen Werte auf ein Minimum - ein Minimum, das innerhalb der Toleranzspanne liegt! — zu verringern. Tab. 9 zeigt die einzelnen Intervalle der vier Skalen plus deren Abweichungen von diesen idealisierten Schritten. 
Tab. 9: Skalen der Xylophone als additive Reihung von Intervallklassen

\begin{tabular}{lrrrrrrr} 
Intervallklassen & $\mathbf{1 2 6 , 0 0}$ & $\mathbf{1 8 9 , 0 0}$ & $\mathbf{2 1 6 , 0 0}$ & & & \\
\hline B 8995 & 185,30 & 211,13 & 122,32 & 320,11 & 213,80 & & \\
Abweichungen & $-3,70$ & $-4,87$ & $-3,68$ & 5,11 & $-2,20$ & & \\
\hline B 9088 & 224,50 & 190,34 & 197,84 & 146,89 & 321,93 & & \\
Abweichungen & 8,50 & 1,34 & 8,84 & 20,89 & 6,93 & & \\
\hline B 9029 & 129,46 & 189,57 & 127,33 & 115,66 & 192,09 & & \\
Abweichungen & 3,46 & 0,57 & 1,33 & $-10,34$ & 3,09 & & \\
\hline B 28284 & 298,58 & 194,36 & 104,05 & 191,04 & 136,58 & 177,62 & 265,84 \\
Abweichungen & $-16,42$ & 5,36 & $-21,95$ & 2,04 & 10,58 & $-11,38$ & 13,84
\end{tabular}

$-113-$

Werte und Reihung der Intervalle wurden aus den obigen Tabellen entnommen. Die Reihenfolge entspricht somit jeweils einer aufsteigenden Skala. Bei Doppelintervallen wurden zwei Standardintervalle subtrahiert: bei 320,11, 321,93 und 298,58 Cents die Intervalle A und B, bei 265,84 Cents zweimal Intervall A.

Alle Abweichungen liegen unter der Toleranzschwelle, Werte von knapp über 20 Cents sind als nur geringfügige Überschreitung der gesetzten Schwelle wohl tolerabel. Besonders auffällig ist, dass die Oktave offenbar nicht als Bezugsrahmen für die Stimmung genützt wird, wie besonders deutlich die Skala des mangolongondo (B 28284) zeigt. Eher ist von einer additiven Reihung auszugehen, bei der die Intervalle in praktisch beliebiger Folge zu einer Skala zusammengefügt werden und folglich verschieden viele Skalentöne innerhalb einer Oktave zu liegen kommen. Zumindest ist aus diesen vier untersuchten Skalen, was die Reihenfolge der drei Intervalle angeht, keine weitere Systematik ablesbar.

Woher eine Stimmung dieser Art kommt, ist wohl nicht zu ermitteln. Eine Ableitung von temperierten Skalen oder von harmonischen Reihen wäre zu vermuten, doch bleiben solche Spekulationen im Bereich von Tautologien: so gut wie jedes Intervall lässt sich als Intervall oder Kombination von Intervallen einer Temperatur oder einer Obertonreihe auffassen. Etwas Spezifisches für diese Skala wäre damit nicht ausgesagt. Dass allerdings die Annahme von verschiedenen Standardintervallen berechtigt ist, zeigen nicht zuletzt die Studien von Dehoux/Voisin (siehe oben), die in Zentralafrika Ähnliches beobachten konnten. Auch die Toleranzspannen treffen sich, wenn sie diese mit 'more or less 15 cents' angeben.

$-116-$

Auf jeden Fall vermag diese Hypothese die Stimmungen der vier Xylophone besser zu beschreiben und zu erklären als eine harmonische Reihe oder eine 
äquidistante Stimmung. Denn während bei letzteren relativ große, letztlich unwahrscheinliche Abweichungen inkauf genommen werden müssen und damit möglicherweise charakteristische Eigenheiten der Skalen als Unregelmäßigkeiten ausgeblendet sind, werden die Varianzen hier als systemimmanent aufgefasst und bleiben die nun anzunehmenden Abweichungen in einem überschaubaren und plausiblen Rahmen.

$-117-$

Bleibt als dritte Gruppe ein einziges Xylophon: das mangolongondo Waisoni Msusas. Hier zeigte sich, ähnlich den beiden dimbila-Xylophonen des Ruvuma-Tals, dass alleine die Hypothese einer äquidistanten Skala als Erklärungsmuster in Anwendung gebracht werden kann. Ein Vergleich mit einer harmonischen Reihe wäre wenig sinnvoll, das Standardintervall für alle Intervallschritte beträgt entsprechend der äquidistanten Hexatonik 200 Cents. Und trotzdem scheint gerade dieses mangolongondo das Instrument des größten Experten des Xylophonstils des Nyasa/Ruvuma-Raums zu sein. Warum also ist das Xylophon nicht nach dem offenbar 'ursprünglichen' Schema von drei Standardintervallen gestimmt? Diese Frage muss offen bleiben. Möglicherweise könnte an islamische Einflüsse gedacht werden, wie Kubik vermutet. ${ }^{18}$

Fazit: Auf die Frage nach den Xylophonstimmungen im Nyasa/RuvumaGebiet gibt es keine eindeutige Antwort. Die untersuchten Instrumente zeigten, dass mehrere Skalen denkbar sind und dass ihnen vielleicht nicht jene große Bedeutung zukommt, wie europäische Forscher es anzunehmen geneigt sind. Vielmehr geht aus der Untersuchung hervor, dass dieselbe Art von Musik auf sehr verschiedenen Skalen gespielt werden kann. Bedeutender als diese sind das Konzept einer Art von Bordun, der durch Interlocking erzeugt wird, und die Vorliebe für Sekundklänge, die nicht auf exakte Intervallgrößen fixiert sind (beispielsweise 129,46 Cents bei B 9029 und 224,50 Cents bei B 9088).

$$
-119-
$$

Als kleiner Wermutstropfen bleibt die Tatsache, dass auch die Basis dieser Studie mit acht verschiedenen Xylophonen nur einen Bruchteil der Aufnahmen zum Xylophonspiel aus dem betreffenden Gebiet abdeckt. Es wäre wünschenswert, könnte diese Lücke durch künftige Forschungen geschlossen werden. An der Feststellung, dass die Struktur der Musik nur einen geringen Zusammenhang mit der tatsächlichen Stimmung der Instrumente aufweist, werden weitere Studien wohl nichts ändern. Ob jedoch die Annahme 
von Intervallklassen haltbar ist, kann nur auf diesem Weg weiter untersucht werden.

Verwendete Literatur:

Bork, I[ngolf]. 1986. Modalanalyse eines Xylophonstabes, in: Fortschritte der Akustik- DAGA '86, 401-404. Bad Honnef: DPG-GmbH.

Dehoux, Dehoux und Frédéric Voisin 1992. Analytic Procedures with Scales in Central African Xylophone Music, in: European Studies in Ethnomusicology: Historical Developments and Recent Trends. Selected papers presented at the VIIth European seminar in ethnomusicology, Berlin, October 1-6, 1990, hrsg. von Max Peter Baumann, Artur Simon, Ulrich Wegner (Intercultural Music Studies 4), 174-188. Wilhelmshaven: Florian Noetzel. 1993. An interactive experimental method for the determination of musical scales in oral cultures. Application to the xylophone music of Central Africa, in: Music and the Cognitive Sciences 1990. Proceedings of Cambridge Conference on Music and the Cognitive Sciences 1990, hrsg. von Ian Cross, Irène Deliège (Contemporary Music Review 9), 1319. New York: Harwood.

Hall, Donald E. 1997. Musikalische Akustik. Ein Handbuch, hrsg. von Johannes Goebel, a. d. Amerikan. von Thomas A. Troge (Veröffentlichungen des Zentrums für Kunst und Medienforschung Karlsruhe, Institut für Musik und Akustik). Mainz [u. a.]: Schott.

Kragl, Florian 2001. Konstruktiver Realismus und musikalische Analyse. Strukturalistische Betrachtungen zu K. 465 von Wolfgang Amadeus Mozart. Online im Internet: Frankfurter Zeitschrift für Musikwissenschaft 4:77-127 (URL: www.fzmw.de).

Kubik, Gerhard 1962. [Protokolle und Originalprotokolle zu den Aufnahmen der Afrikaexpedition 1962 nach Moçambique], archiviert im Phonogrammarchiv der Österreichischen Akademie der Wissenschaften (Wien) als B 8993-B 9158.

1964. Recording and Studying Music in Northern Moçambique, in: African Music 3,3:77-100. Corrigenda in: African Music 4,4 (1970):136137.

1965. Transcription of Mangwilo Xylophone Music from Film Strips, in: African Music 3,4:35-51. Corrigenda in: African Music 4,4 (1970):137.

1967. [Protokolle und Originalprotokolle zu den Aufnahmen der Afrikaexpedition 1967 nach Malawi], archiviert im Phonogrammarchiv der 
Österreichischen Akademie der Wissenschaften (Wien) als B 13420B13539.

1982. Ostafrika (Musikgeschichte in Bildern I, 10). Leipzig: VEB Deutscher Verlag für Musik.

1983. Die Amadinda-Musik von Buganda, in: Musik in Afrika, hrsg. von Artur Simon (Veröffentlichungen des Museums für Völkerkunde Berlin, N.F. 40; Abt. Musikethnologie IV), 139-165. Berlin: Museum für Völkerkunde.

1983/84. [Protokolle und Originalprotokolle zu den Aufnahmen der Afrikaexpedition 1983/84 nach Malawi], archiviert im Phonogrammarchiv der Österreichischen Akademie der Wissenschaften (Wien) als B $28278 \mathrm{ff}$.

1987. Malawian Music. A Framework for Analysis. Zomba (Malawi): University of Malawi.

1989. Opeka nyimbo. Musiker-Komponisten aus dem südlichen Malawi. [2 LPs] (Museum Collection Berlin [West] 15). Berlin: Museum für Völkerkunde. [Zitiert wird das Begleitheft.]

1992. Embaire Xylophone Music of Samusiri Babalanda (Uganda 1968), in: The World of Music 34,1:57-84.

2001. Mangwilo - Analyse einer denkwürdigen HolmxylophonTradition in Nord-Moçambique, 1962. Manuskript, Masch. [1+73 S. paginiert als 0 (Deckblatt) und 1-73, zit. nach dieser Zählung. Abgesehen von wenigen sprachlichen Verbesserungen unveränderte Fassung einer Studie aus der Mitte der 1980er Jahre. Die Arbeit soll in einer der nächsten Ausgaben der Musicologica Austriaca erscheinen.]

Schmidhofer, August 1994. Präzisionsmessungen an Xylophonstäben, in: Vergleichend-systematische Musikwissenschaft. Beiträge zu Methode und Problematik der systematischen, ethnologischen und historischen Musikwissenschaft. Franz Födermayr zum 60. Geburtstag, hrsg. von Elisabeth Th. Hilscher, Theophil Antonicek (Wiener Veröffentlichungen zur Musikwissenschaft 31), 327-335. Tutzing: Hans Schneider.

Terhardt, Ernst 1998. Akustische Kommunikation. Grundlagen mit Hörbeispielen. Berlin [u. a.]: Springer.

van Zanten, Wim 1980. The equidistant heptatonic scale of the Asena in Malawi, in: African Music 6,1:107-125.

Zwicker, E[rnst] und Hugo Fastl 1990. Psychoacoustics. Facts and Models. Berlin [u. a.]: Springer. 